\title{
铂催化氧杂二环烯烃与芳基磺酰肼的顺式-立体控制开环反应
}

\author{
王 林 ${ }^{\dagger, a, c}$ \\ 杨丽 $\overline{\text { 网 }}{ }^{\dagger}, a$ \\ 欧云付 $a$ \\ 徐石海*, $a, b$ \\ 林啟福 $c$ \\ 杨定乔*,c \\ ( ${ }^{a}$ 暨南大学分析测试中心 广州 510632) \\ ( ${ }^{b}$ 暨南大学化学与材料学院 广州 510632) \\ ( $c$ 华南师范大学化学学院 广州 510006)
}

\begin{abstract}
摘要 开发了一种铂催化氧杂二环烯烃与芳基磺酰肼的顺式-立体控制开环反应. 该方法显示出高效性和良好的官能 团耐受性，在温和条件下能以高达 89\%的产率得到顺式-2-芳基-1,2-二氢菜-1-醇(3)或脱水产物 2-芳基荎(4). 此外，通过 $\mathrm{X}$ 射线单晶衍射分析确认了产物( $1 S^{*}, 2 R^{*}$ )-6,7-二溴-2-(4-甲基苯基)-1,2-二氢蒜-1-醇(3db)的顺式-1,2-构型. 根据实验结 果, 提出了一种合理的开环反应机理. 值得注意的是, 在开环反应中, 芳基磺酰肼通过释放 $\mathrm{N}_{2}$ 和 $\mathrm{SO}_{2}$ 形成碳负离子作 为亲核试剂.
\end{abstract}

关键词 铂催化剂; 开环; 氧杂二环烯烃; 芳基磺酰肼; 亲核试剂

\section{Platinum-Catalyzed syn-Stereocontrolled Ring-Opening of Oxabicyclic Alkenes with Arylsulfonyl Hydrazides}

\author{
Wang, Lin ${ }^{\dagger, a, c} \quad$ Yang, Lili ${ }^{\dagger, a} \quad$ Ou, Yunfu ${ }^{a} \quad$ Xu, Shihai ${ }^{*, a, b}$ \\ Lin, Qifu ${ }^{c} \quad$ Yang, Dingqiao $*, c$ \\ ( ${ }^{a}$ Analytical and Testing Center, Jinan University, Guangzhou 510632) \\ ( ${ }^{b}$ College of Chemistry and Materials Science, Jinan University, Guangzhou 510632) \\ ( ${ }^{c}$ School of Chemistry, South China Normal University, Guangzhou 510006)
}

\begin{abstract}
A platinum-catalyzed syn-stereocontrolled ring-opening reaction of oxabicyclic alkenes with arylsulfonyl hydrazides was developed. This protocol exhibited high efficiency and good functional group tolerance, affording cis-2-aryl-1,2dihydronaphthalen-1-ols (3) or 2-aryl-naphthalenes (4) as dehydrated products in good to excellent yields under mild conditions (up to $89 \%$ ). In addition, the $c i s$-1,2-configuration of product $\left(1 S^{*}, 2 R^{*}\right)$-6,7-dibromo-2-( $p$-tolyl)-1,2-dihydronaphthalen1-ol (3db) was confirmed by X-ray single crystal diffraction analysis. Based on the results, a plausible mechanism for the ring-opening reaction was proposed. Remarkably, arylsulfonyl hydrazides were used as carboanion nucleophiles in the ring-opening reaction via releasing $\mathrm{N}_{2}$ and $\mathrm{SO}_{2}$ in situ.
\end{abstract}

Keywords platinum catalyst; ring-opening; oxabicyclic alkenes; arylsulfonyl hydrazides; nucleophiles

\section{Introduction}

The transition metal-catalyzed ring-opening reaction of oxa- and aza-bicyclic alkenes is a useful method to construct carbon-carbon and carbon-heteroatom bonds. ${ }^{[1]}$ It is well known that 1,2-dihydronaphthalene skeleton was found in a wide range of naturally occurring compounds which exhibited a variety of biological activities. ${ }^{[2]}$ In addi- tion, this transformation is valuable because multiple stereocenters can be established in a single step. In this regard, numerous metal catalysts, including $\mathrm{Ir}^{[3]} \mathrm{Ni}^{[4]} \mathrm{Pd},{ }^{[5]}$ $\mathrm{Cu},{ }^{[6]} \mathrm{Rh},{ }^{[7]} \mathrm{Pt},{ }^{[8]} \mathrm{Ru},{ }^{[9]} \mathrm{Fe},{ }^{[10]}$ and so forth, ${ }^{[11]}$ have been investigated for the ring-opening reaction of heterobicyclic alkenes with various carbon and heteroatom nucleophiles.

Since the seminal work of Lautens, ${ }^{[1 \mathrm{~b}, 1 \mathrm{~d}]}$ many research

\footnotetext{
* Corresponding authors. E-mail: txush@jnu.edu.cn; yangdq@scnu.edu.cn

Received June 30, 2020; revised July 23, 2020; published online August 18, 2020.

Project supported by the National Natural Science Foundation of China (Nos. 21672084, 21372090) and the Special Fund Project of Department of Natural Resources of Guangdong Province (Guangdong Natural Resources Cooperation) (No. 2020037).

国家自然科学基金(Nos. 21672084, 21372090)和广东省自然资源厅专项资金(粤自然资合) (No. 2020037)资助项目.

共同第一作者(These authors contributed equally to this work).
} 
groups have been working on the transition metalcatalyzed asymmetric ring-opening (ARO) reactions of oxa(aza)bicyclic alkenes and make some considerable progress. Our group has long-term interest in the ARO reactions of oxa- and aza-bicyclic alkenes with various nucleophiles. The nucleophiles, such as amines, ${ }^{[3,3 c, 3 e, 12]}$ phenols, ${ }^{[8 \mathrm{a}, 13]}$ alcohols, ${ }^{[3 \mathrm{f}, 14]}$ carboxylic acids, ${ }^{[3 \mathrm{~b}, 3 \mathrm{~d}]}$ Grignard reagents ${ }^{[15]}$ and organic zincs ${ }^{[16]}$ have been found as feasible nucleophiles used in the ring-opening reaction. In recent years, arylboronic acids and arylsulfinate salts, because of their stability and low toxicity, also have been employed as effective carbanion nucleophiles in ringopening reactions of oxabicyclic alkenes to afford the corresponding product 2-aryl-1,2-dihydronaphthalen-1-ols in high yields. For instance, our group demonstrated platinum-catalyzed ring-opening of oxabenzonorbornadienes with sodium sulfinates ${ }^{[17]}$ and arylboronic acids ${ }^{[18]}$, which generated cis-1,2-ring-opening products in high yields.

Arylsulfonyl hydrazides are generally stable in the air. They can be prepared in one step from readily available arylsulfonyl chlorides and hydrazine hydrates, ${ }^{[19]}$ and increasing attention has recently been paid to the construction of $\mathrm{C}-\mathrm{C}$ bonds through the extrusion of $\mathrm{N}_{2}$ and $\mathrm{SO}_{2}$ in situ. $^{[20]}$ Recently, our group ${ }^{[21]}$ has reported palladium-catalyzed syn-stereocontrolled ring-opening reactions of oxabenzonorbornadienes with arylsulfonyl hydrazides, affording the desired products in good to excellent yields (up to $96 \%$ ) under an air atmosphere. To the best of our knowledge, there is no literature reported about platinum-catalyzed syn-stereocontrolled ring-opening of oxabicyclic alkenes with arylsulfonyl hydrazides till now. Our continuous interest in this type of reactions promotes us to further explore and expand the possibility of this reaction in the presence of platinum catalyst. Herein, we reported platinum-catalyzed ring-opening of oxabicyclic alkenes with arylsulfonyl hydrazides to afford the corresponding products of cis-2-aryl-1,2-dihydronapthalene-1-ols and 2-aryl-napthalenes in good to excellent yields.

\section{Results and discussion}

Oxabenzonorbornadiene (1a) and phenylsulfonyl hydrazide (2a) were initially chosen as model substrates to identify the feasibility of our process. When $\mathbf{2 a}$ reacted with $\mathbf{1 a}$ in 1,2-dichloroethane (DCE) at $80{ }^{\circ} \mathrm{C}$ in the presence of $\mathrm{PtCl}_{2}(10 \mathrm{~mol} \%), \mathrm{PPh}_{3}(20 \mathrm{~mol} \%)$ and $\mathrm{Cu}(\mathrm{OAc})_{2}$ (3 equiv.), the desired ring-opening product 3aa was obtained in $62 \%$ yield after $4 \mathrm{~h}$ (Table 1, Entry 1 ). Other two platinum catalysts were then screened, and $\mathrm{PtCl}_{2}$ was the best one (Entries $1 \sim 3$ ). It was worth mentioning that the ring-opening reaction did not take place in the absence of $\mathrm{PtCl}_{2}$ (Entry 4). When the catalyst loading was $5 \mathrm{~mol} \%$ and $20 \mathrm{~mol} \%$, the ring-opening product 3 aa could be obtained with yields of $39 \%$ and $60 \%$, respectively (Entries 5, 6). In order to further improve the yield of 3aa, series achiral phosphine ligands, including 1,3-bis(diphenylphosphino)propane (DPPP), 1,2-bis(diphenylphosphino)ethane
(DPPE) and 1,1'-bis(diphenylphosphino)ferrocene (DPPF) were examined for the ring-opening reaction (Entries $7 \sim$ 9) (yields up to $62 \%$ ). Moreover, chiral phosphine ligands, including $(S)$-SEGPHOS, $(R, S)$-PPF- ${ }^{t} \mathrm{Bu}_{2},(R)$-BINAP, $(S)$ DM-SEGPHOS, $(4 S, 5 S)$-DIOP and $(2 S, 4 S)$-BDPP were also tested. Unfortunately, the product 3aa was obtained only in moderate yield and low enantioselectivity (up to $53 \%$ yield and 43\% ee) (Entries 10 15). Therefore, among the ligands screened, achiral ligand $\mathrm{PPh}_{3}$ was the most effective in terms of yield (Entries $1 \sim 15$ ). Next, several cupric salts, such as $\mathrm{CuO}, \mathrm{CuCl}_{2}$ and $\mathrm{Cu}(\mathrm{acac})_{2}$ were also investigated. Unfortunately, all of them gave low yields (Entries 16 18). The effect of solvent was then examined (Entries 1, 19 21). The results indicated that solvents played an important role in the ring-opening reaction. The solvent DCE was the optimal solvent. Furthermore, the impact of reaction temperature on the reaction efficiency was investigated (Entries 22 23). When the reaction temperature was 60 or $100{ }^{\circ} \mathrm{C}$, the product 3aa was obtained in moderate yield $(53 \%$ and $48 \%$, respectively). At last, the impact of the amount of $\mathrm{Cu}(\mathrm{OAc})_{2}$ on the reaction was investigated (Entries 24 26). The results indicated that 1.5 equiv. of $\mathrm{Cu}(\mathrm{OAc})_{2}$ gave the best yield $(82 \%)$. It should be noted that in the absence of $\mathrm{Cu}(\mathrm{OAc})_{2}$, none of the targeted product was observed (Entry 27). Meanwhile, product 3aa could be obtained in $20 \%$ yield in the absence of $\mathrm{PPh}_{3}$ (Entry 28). Therefore, the optimal reaction conditions were: $10 \mathrm{~mol} \% \mathrm{PtCl}_{2}, 20 \mathrm{~mol} \% \mathrm{PPh}_{3}, 1.5$ equiv. of $\mathrm{Cu}(\mathrm{OAc})_{2}$ in $\mathrm{DCE}$ at $80{ }^{\circ} \mathrm{C}$ for $4 \mathrm{~h}$.

Next, attention was paid to the ring-opening reaction of 1 a with a variety of arylsulfonyl hydrazides under the optimized reaction conditions (Table 2). In general, all of arylsulfonyl hydrazides $\mathbf{2} \mathbf{a} \sim \mathbf{2 h}$ as nucleophiles could react smoothly with 1a and provided the corresponding targeted products 3aa $3 \mathbf{3 h}$ in moderate to good yields (52\% $85 \%$ ). The substituents attached on arylsulfonyl hydrazides had obvious electronic effect on the ring-opening reaction. Compared with arylsulfonyl hydrazides with strong electron donating groups, the ring opening reaction of arylsulfonylhydrazides with electron withdrawing groups on benzene ring was better. Arylsulfonyl hydrazides $\mathbf{2 e} \sim \mathbf{2 g}$ bearing electron-withdrawing groups could react smoothly with $\mathbf{1 a}$ and afforded the corresponding products $\mathbf{3 a e} \sim \mathbf{3 a g}$ in good yields $(78 \%, 85 \%$ and $77 \%$, respectively). Arylsulfonyl hydrazides $\mathbf{2 b} \sim \mathbf{2 c}$ bearing electron-donating groups could react with 1a and afforded the corresponding products 3ab $\sim 3 a c$ in moderate yields $(68 \%$ and $66 \%$, respectively). 2-Chlorobenzenesulfonyl hydrazide (2d) could react with $1 \mathbf{a}$ and afforded ring-opening product $\mathbf{3 a d}$ in $60 \%$ yield. In addition to these, when 2,4,6-trimethylbenzenesulfonyl hydrazide (2h) as nucleophile was employed in the reaction, the desired product 3ah was obtained in $52 \%$ yield, which probably due to steric hindrance.

The scope of this ring-opening reaction was further expanded to various oxabenzonorbornadienes $\mathbf{1 b} \sim \mathbf{1 f}$. The results showed that the reaction of various oxabenzonor- 
Table 1 Optimization for the reaction conditions ${ }^{a}$
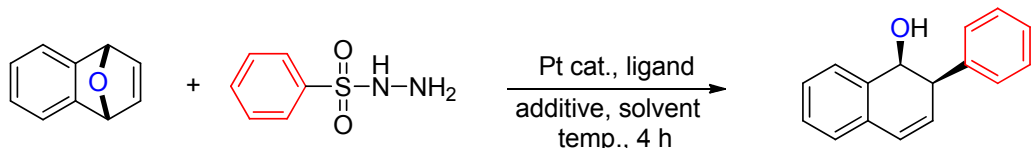

$1 \mathrm{a}$

$2 \mathrm{a}$

3aa

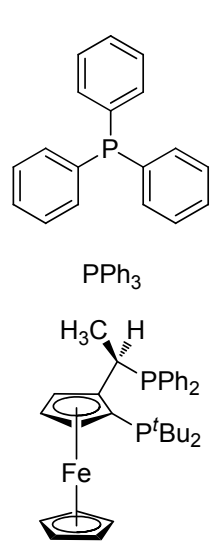

$(R, S)-P P F-P^{t} \mathrm{Bu}_{2}$<smiles>c1ccc(P(CCP(c2ccccc2)c2ccccc2)c2ccccc2)cc1</smiles>

DPPP<smiles>Pc1ccc2ccccc2c1-c1c(P(c2ccccc2)c2ccccc2)ccc2ccccc12</smiles>

(R)-BINAP

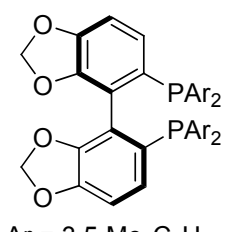

$\mathrm{Ar}=3,5-\mathrm{Me}_{2} \mathrm{C}_{6} \mathrm{H}_{3}$ (S)-DM-SEGPHOS
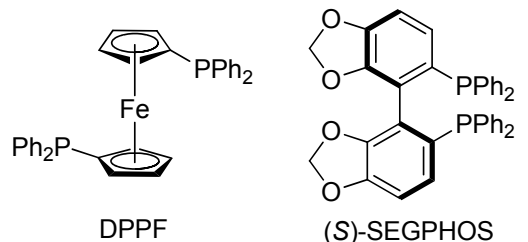

(S)-SEGPHOS<smiles>CC1(C)O[C@H](CP)[C@H](CP)O1</smiles>

(4S,5S)-DIOP<smiles>CC(C[C@H](C)Pc1ccccc1)Pc1ccccc1</smiles>

(2S,4S)-BDPP

\begin{tabular}{|c|c|c|c|c|c|c|}
\hline Entry & Pt catalyst (mol\%) & Ligand (mol\%) & Additive (equiv.) & Solvent & Yield $^{b} / \%$ & $e e^{c} / \%$ \\
\hline 1 & $\mathrm{PtCl}_{2}(10)$ & $\mathrm{PPh}_{3}(20)$ & $\mathrm{Cu}(\mathrm{OAc})_{2}(3)$ & DCE & 62 & \\
\hline 2 & $\mathrm{Pt}(\mathrm{COD}) \mathrm{Cl}_{2}(10)$ & $\mathrm{PPh}_{3}(20)$ & $\mathrm{Cu}(\mathrm{OAc})_{2}(3)$ & DCE & 58 & \\
\hline 3 & $\operatorname{Pt}(\mathrm{acac})_{2}(10)$ & $\mathrm{PPh}_{3}(20)$ & $\mathrm{Cu}(\mathrm{OAc})_{2}(3)$ & DCE & 36 & \\
\hline 4 & - & $\mathrm{PPh}_{3}(20)$ & $\mathrm{Cu}(\mathrm{OAc})_{2}(3)$ & DCE & 0 & \\
\hline 5 & $\mathrm{PtCl}_{2}(5)$ & $\mathrm{PPh}_{3}(10)$ & $\mathrm{Cu}(\mathrm{OAc})_{2}(3)$ & DCE & 39 & \\
\hline 6 & $\mathrm{PtCl}_{2}(20)$ & $\mathrm{PPh}_{3}(40)$ & $\mathrm{Cu}(\mathrm{OAc})_{2}(3)$ & DCE & 60 & \\
\hline 7 & $\mathrm{PtCl}_{2}(10)$ & DPPP & $\mathrm{Cu}(\mathrm{OAc})_{2}(3)$ & DCE & Trace & \\
\hline 8 & $\mathrm{PtCl}_{2}(10)$ & DPPE & $\mathrm{Cu}(\mathrm{OAc})_{2}(3)$ & DCE & Trace & \\
\hline 9 & $\mathrm{PtCl}_{2}(10)$ & DPPF & $\mathrm{Cu}(\mathrm{OAc})_{2}(3)$ & DCE & 41 & \\
\hline 10 & $\mathrm{PtCl}_{2}(10)$ & $(S)$-SEGPHOS & $\mathrm{Cu}(\mathrm{OAc})_{2}(3)$ & DCE & 53 & 36 \\
\hline 11 & $\mathrm{PtCl}_{2}(10)$ & $(R, S)-\mathrm{PPF}-{ }^{t} \mathrm{Bu}_{2}$ & $\mathrm{Cu}(\mathrm{OAc})_{2}(3)$ & DCE & 36 & 20 \\
\hline 12 & $\mathrm{PtCl}_{2}(10)$ & $(R)$-BINAP & $\mathrm{Cu}(\mathrm{OAc})_{2}(3)$ & DCE & 48 & 10 \\
\hline 13 & $\mathrm{PtCl}_{2}(10)$ & $(S)$-DM-SEGPHOS & $\mathrm{Cu}(\mathrm{OAc})_{2}(3)$ & DCE & 50 & 43 \\
\hline 14 & $\mathrm{PtCl}_{2}(10)$ & $(4 S, 5 S)-\mathrm{DIOP}$ & $\mathrm{Cu}(\mathrm{OAc})_{2}(3)$ & DCE & 10 & 5 \\
\hline 15 & $\mathrm{PtCl}_{2}(10)$ & $(2 S, 4 S)-\mathrm{BDPP}$ & $\mathrm{Cu}(\mathrm{OAc})_{2}(3)$ & DCE & 8 & 5 \\
\hline 16 & $\mathrm{PtCl}_{2}(10)$ & $\mathrm{PPh}_{3}$ & $\mathrm{CuO}(3)$ & DCE & Trace & \\
\hline 17 & $\mathrm{PtCl}_{2}(10)$ & $\mathrm{PPh}_{3}$ & $\mathrm{CuCl}_{2}(3)$ & DCE & 23 & \\
\hline 18 & $\mathrm{PtCl}_{2}(10)$ & $\mathrm{PPh}_{3}$ & $\mathrm{Cu}(\mathrm{acac})_{2}(3)$ & DCE & 52 & \\
\hline 19 & $\mathrm{PtCl}_{2}(10)$ & $\mathrm{PPh}_{3}$ & $\mathrm{Cu}(\mathrm{OAc})_{2}(3)$ & 1,4-Dioxane & 46 & \\
\hline 20 & $\mathrm{PtCl}_{2}(10)$ & $\mathrm{PPh}_{3}$ & $\mathrm{Cu}(\mathrm{OAc})_{2}(3)$ & THF & 51 & \\
\hline 21 & $\mathrm{PtCl}_{2}(10)$ & $\mathrm{PPh}_{3}$ & $\mathrm{Cu}(\mathrm{OAc})_{2}(3)$ & DMF & 37 & \\
\hline $22^{d}$ & $\mathrm{PtCl}_{2}(10)$ & $\mathrm{PPh}_{3}$ & $\mathrm{Cu}(\mathrm{OAc})_{2}(3)$ & DCE & 53 & \\
\hline $23^{e}$ & $\mathrm{PtCl}_{2}(10)$ & $\mathrm{PPh}_{3}$ & $\mathrm{Cu}(\mathrm{OAc})_{2}(3)$ & DCE & 48 & \\
\hline 24 & $\mathrm{PtCl}_{2}(10)$ & $\mathrm{PPh}_{3}$ & $\mathrm{Cu}(\mathrm{OAc})_{2}(2)$ & DCE & 73 & \\
\hline 25 & $\mathrm{PtCl}_{2}(10)$ & $\mathrm{PPh}_{3}$ & $\mathrm{Cu}(\mathrm{OAc})_{2}(1.5)$ & DCE & 82 & \\
\hline 26 & $\mathrm{PtCl}_{2}(10)$ & $\mathrm{PPh}_{3}$ & $\mathrm{Cu}(\mathrm{OAc})_{2}(1)$ & DCE & 65 & \\
\hline 27 & $\mathrm{PtCl}_{2}(10)$ & $\mathrm{PPh}_{3}$ & - & DCE & n.r. & \\
\hline 28 & $\mathrm{PtCl}_{2}(10)$ & 一 & $\mathrm{Cu}(\mathrm{OAc})_{2}(1.5)$ & DCE & 20 & \\
\hline
\end{tabular}

${ }^{a}$ The reaction was carried out with Pt catalyst, ligand, additive, 1a $(0.1 \mathrm{mmol}, 1.0$ equiv. $)$ and $\mathbf{2 a}(0.2 \mathrm{mmol}, 2.0$ equiv. $)$ in the corresponding solvent at $80{ }^{\circ} \mathrm{C}$ under an air atmosphere for $4 \mathrm{~h} .{ }^{b}$ Isolated yield. ${ }^{c}$ Determined by HPLC with a Chiralcel OD-H column. ${ }^{d} 60{ }^{\circ} \mathrm{C} .{ }^{e} 100{ }^{\circ} \mathrm{C}$.

bornadienes with arylsulfonyl hydrazides proceeded smoothly to afford corresponding target product in moderate to excellent yields (Table 3). The ring-opening reactions of oxabenzonorbornadienes $\mathbf{1 d} \sim \mathbf{1 f}$ with various ar- ylsulfonyl hydrazides proceeded smoothly to afford the expected products with good to excellent yields (up to $89 \%$ ). Oxabenzonorbornadienes $\mathbf{1 d}$ and $\mathbf{1 f}$ bearing bromine and fluorine groups showed better reaction efficiency than 
Table 2 Platinum-catalyzed ring-opening of oxabenzonorbornadiene 1a with various arylsulfonyl hydrazides $\mathbf{2 a} \sim \mathbf{2 h}^{a}$<smiles>NN[14C](=O)[14C]1=[14CH][14CH]=[14C]2O[C@H]1c1ccccc12</smiles>

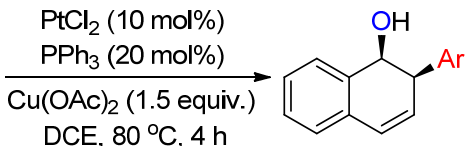
1a $2 a \sim 2 h$ DCE $80^{\circ} \mathrm{C}, 4 \mathrm{~h}$ 3aa 3ah

\begin{tabular}{clcc}
\hline Entry & \multicolumn{1}{c}{$\mathrm{Ar}$} & Product & Yield $^{b} \%$ \\
\hline 1 & $\mathrm{C}_{6} \mathrm{H}_{5}$ & 3aa & 82 \\
2 & $4-\mathrm{CH}_{3} \mathrm{C}_{6} \mathrm{H}_{4}$ & 3ab & 68 \\
3 & $4-\mathrm{CH}_{3} \mathrm{OC}_{6} \mathrm{H}_{4}$ & $\mathbf{3 a c}$ & 66 \\
4 & $2-\mathrm{ClC}_{6} \mathrm{H}_{4}$ & 3ad & 60 \\
5 & $4-\mathrm{ClC}_{6} \mathrm{H}_{4}$ & 3ae & 78 \\
6 & $4-\mathrm{FC}_{6} \mathrm{H}_{4}$ & 3af & 85 \\
7 & $4-\mathrm{O}_{2} \mathrm{NC}_{6} \mathrm{H}_{4}$ & 3ag & 77 \\
8 & $2,4,6-\left(\mathrm{CH}_{3}\right)_{3} \mathrm{C}_{6} \mathrm{H}_{2}$ & 3ah & 52 \\
\hline
\end{tabular}

${ }^{a}$ Conditions: 1a $(0.1 \mathrm{mmol}, 14.4 \mathrm{mg}), 2(0.2 \mathrm{mmol}), \mathrm{Cu}(\mathrm{OAc})_{2}(1.5$ equiv. $)$, $\mathrm{PtCl}_{2}(10 \mathrm{~mol} \%), \mathrm{PPh}_{3}(20 \mathrm{~mol} \%), \mathrm{DCE}(2 \mathrm{~mL}), 80{ }^{\circ} \mathrm{C} .{ }^{b}$ Isolated yield.

Table 3 Platinum-catalyzed ring-opening of oxabenzonorbornadienes $\mathbf{1 b} \sim \mathbf{1 g}$ with various arylsulfonyl hydrazides ${ }^{a}$

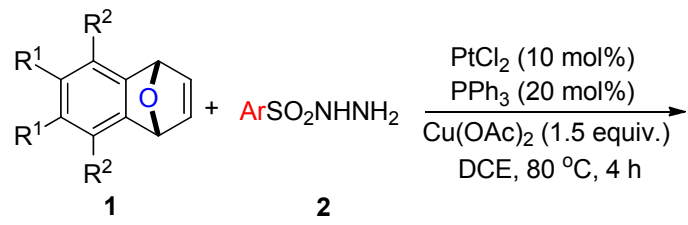

1b: $\mathrm{R}^{1}=\mathrm{H}, \mathrm{R}^{2}=\mathrm{OCH}_{3} ; \mathbf{1 c}: \mathrm{R}^{1}=\mathrm{OCH}_{3}, \mathrm{R}^{2}=\mathrm{H} ; \mathbf{1 d}: \mathrm{R}^{1}=\mathrm{Br}, \mathrm{R}^{2}=\mathrm{H}$; 1e: $R^{1}=\mathrm{CH}_{3}, \mathrm{R}^{2}=\mathrm{H} ; \mathbf{1 f}: \mathrm{R}^{1}=\mathrm{F}, \mathrm{R}^{2}=\mathrm{H} ; \mathbf{1 g}: \mathrm{R}^{1}+\mathrm{R}^{2}=$<smiles>[R]c1c([R])c([R])c2c(c1[R])C=CC([Al])C2O</smiles>

\begin{tabular}{|c|c|c|c|c|c|c|}
\hline Entry & 1 & $\mathrm{Ar}$ & 3 & Yield $^{b} / \%$ of $\mathbf{3}$ & 4 & Yield ${ }^{b} / \%$ of 4 \\
\hline 1 & $1 \mathbf{b}$ & $\mathrm{C}_{6} \mathrm{H}_{5}$ & & & $4 \mathrm{ba}$ & 68 \\
\hline 2 & $1 \mathrm{~b}$ & 4- $\mathrm{CH}_{3} \mathrm{C}_{6} \mathrm{H}_{4}$ & & & $4 \mathrm{bb}$ & 66 \\
\hline 3 & $1 \mathrm{~b}$ & $4-\mathrm{FC}_{6} \mathrm{H}_{4}$ & & & $4 \mathrm{bf}$ & 78 \\
\hline 4 & & $\mathrm{C}_{6} \mathrm{H}_{5}$ & & & $4 \mathrm{ca}$ & 70 \\
\hline 5 & 1c & 4- $\mathrm{CH}_{3} \mathrm{C}_{6} \mathrm{H}_{4}$ & & & $4 \mathrm{cb}$ & 72 \\
\hline 6 & 1d & $\mathrm{C}_{6} \mathrm{H}_{5}$ & 3da & 83 & & \\
\hline 7 & 1d & 4- $\mathrm{CH}_{3} \mathrm{C}_{6} \mathrm{H}_{4}$ & $3 d b$ & 80 & & \\
\hline 8 & 1d & $4-\mathrm{FC}_{6} \mathrm{H}_{4}$ & $3 d f$ & 88 & & \\
\hline 9 & $1 e$ & $\mathrm{C}_{6} \mathrm{H}_{5}$ & 3 ea & 71 & & \\
\hline 10 & 1e & $4-\mathrm{ClC}_{6} \mathrm{H}_{4}$ & 3ee & 72 & & \\
\hline 11 & 1e & $4-\mathrm{O}_{2} \mathrm{NC}_{6} \mathrm{H}_{4}$ & 3 eg & 70 & & \\
\hline 12 & & $\mathrm{C}_{6} \mathrm{H}_{5}$ & $3 f a$ & 87 & & \\
\hline 13 & 1f & 4- $\mathrm{CH}_{3} \mathrm{C}_{6} \mathrm{H}_{4}$ & $3 \mathrm{fb}$ & 80 & & \\
\hline 14 & & $4-\mathrm{FC}_{6} \mathrm{H}_{4}$ & $3 \mathbf{f f}$ & 89 & & \\
\hline 15 & $1 \mathrm{~g}$ & $\mathrm{C}_{6} \mathrm{H}_{5}$ & 3 ga & 52 & & \\
\hline
\end{tabular}

${ }^{a}$ Conditions: $1(0.1 \mathrm{mmol}, 14.4 \mathrm{mg}), 2(0.2 \mathrm{mmol}), \mathrm{Cu}(\mathrm{OAc})_{2}(1.5$ equiv. $)$, $\mathrm{PtCl}_{2}(10 \mathrm{~mol} \%), \mathrm{PPh}_{3}(20 \mathrm{~mol} \%)$, DCE $(2 \mathrm{~mL}), 80{ }^{\circ} \mathrm{C} .{ }^{b}$ Isolated yield.

1e bearing methyl group. Besides, bulkier substrate $\mathbf{1 g}$ afforded product 3ga in 52\% yield, indicating that steric hindrance on substrate $\mathbf{1 g}$ had a negative impact on this ring-opening transformation. Interestingly, the corresponding products 2 -arylnaphthalenes $\mathbf{4 b a} \sim \mathbf{4} \mathbf{c b}$ could be obtained in good yields (up to $78 \%$ ) rather than ringopening products 3 when methoxy substituted oxabenzonorbornadienes $\mathbf{1 b} \sim \mathbf{1 c}$ were used to react with various arylsulfonyl hydrazides.

The cis-1,2-configuration of compound $\mathbf{3 d b}$ was undoubtedly confirmed by X-ray single crystal diffraction analysis (Figure 1). The single crystal was obtained by diffusion method from a mixed solvent of petroleum ether and ethyl acetate.<smiles>Cc1ccc([C@H]2C=Cc3cc(Br)c(Br)cc3[C@H]2O)cc1</smiles>

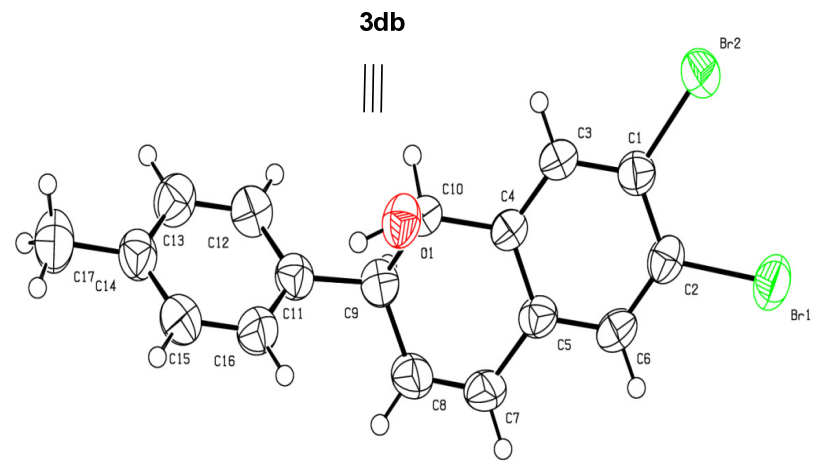

Figure 1 X-ray single crystal diffraction analysis of compound $3 \mathbf{d b}$

To further evaluate the reaction effect on the scale of preparation, scaled-up reaction was then conducted under the current standard conditions. The desired product $\mathbf{3}$ aa was obtained in $68 \%$ yield $(0.302 \mathrm{~g})$, indicating the application of this method in synthetic chemistry (Scheme 1).

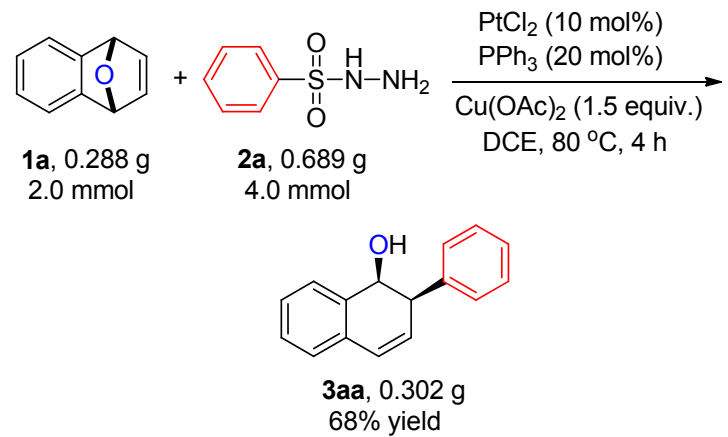

Scheme 1 Scaled-up experiment of $\mathbf{3 a a}$

According to our experimental results and the general mechanism of desulfurization coupling reactions, ${ }^{[20]}$ a plausible mechanism for this ring-opening reaction is proposed (Scheme 2). Initially, ligand $\mathrm{PPh}_{3}$ reacts with $\mathrm{PtCl}_{2}$ to give the active catalyst $\mathrm{Pt}\left(\mathrm{PPh}_{3}\right)_{2} \mathrm{Cl}_{2}$. Then $\mathrm{Pt}\left(\mathrm{PPh}_{3}\right)_{2} \mathrm{Cl}_{2}$ is treated with arylsulfonyl hydrazide 2 to result in complex $\mathbf{A}$, which undergoes $\beta$-hydride elimination to give sulfonyl diazene $\mathbf{B}$ and release $\operatorname{Pt}\left(\mathrm{PPh}_{3}\right)_{2} . \operatorname{Pt}(0)\left(\mathrm{PPh}_{3}\right)_{2}$ is then oxidized by $\mathrm{Cu}(\mathrm{OAc})_{2}$ or $\mathrm{O}_{2}$ from air to regenerate the 


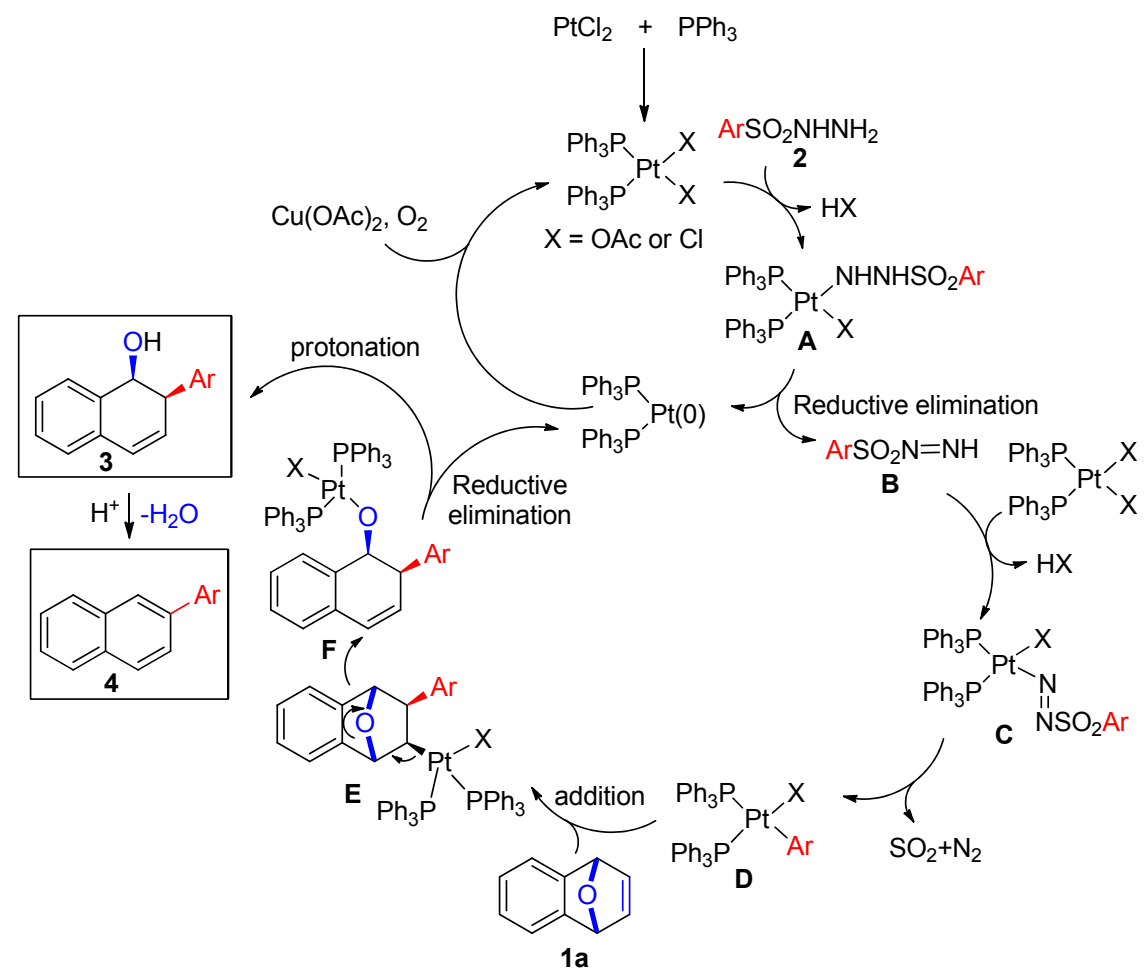

Scheme 2 Plausible mechanism

active catalyst $\mathrm{Pt}\left(\mathrm{PPh}_{3}\right)_{2} \mathrm{Cl}_{2}\left[\mathrm{Pt}\left(\mathrm{PPh}_{3}\right)_{2} \mathrm{X}_{2}\right]$. Meanwhile, one of chlorine atom of $\mathrm{Pt}\left(\mathrm{PPh}_{3}\right)_{2} \mathrm{Cl}_{2}$ is replaced by sulfonyl diazene $\mathbf{B}$ to give intermediate $\mathbf{C}$. Intermediate $\mathbf{C}$ releases nitrogen and sulfur dioxide, affording arylplatinum $\mathbf{D}$, which then undergoes exo-1,2-addition with substrate 1a to yield intermediate $\mathbf{E}$. $\beta$-Oxygen atom elimination of intermediate $\mathbf{E}$ occurs and provides new $\mathrm{Pt}$ intermediate $\mathbf{F}$. Protonation of the intermediate $\mathbf{F}$ affords product $\mathbf{3}$ and a $\mathrm{Pt}\left(\mathrm{PPh}_{3}\right)_{2}$. Further dehydration of $\mathbf{3}$ gives 2-arylnaphthalene 4.

\section{Conclusions}

In summary, an efficient platinum-catalyzed syn-stereocontrolled ring-opening of oxabicyclic alkenes with arylsulfonyl hydrazides via the release of $\mathrm{N}_{2}$ and $\mathrm{SO}_{2}$ in situ under mild conditions has been developed. This synthetic protocol provides a useful tool for the construction of cis-2-aryl-1,2-dihydronaphthalen-1-ols $\mathbf{3}$ and 2-arylnaphthalenes 4 in good to excellent yields. The different substituents of oxabicyclic alkenes play a crucial role in the control of targeted product. The electron-withdrawing groups in the substrate are advantageous for the ringopening reaction, and the dehydrated compounds are more readily available when oxabenzonorbornadienes bearing a strong donor group were used. Further studies toward the evaluating of the biological and pharmaceutical activities of these target compounds are going on in our laboratory.

\section{Experimental section}

\subsection{General information}

All ${ }^{1} \mathrm{H}$ NMR and ${ }^{13} \mathrm{C}$ NMR spectra were recorded at 600 and $150 \mathrm{MHz}$ respectively, using $\mathrm{CDCl}_{3} / \mathrm{CD}_{3} \mathrm{OD}$ as the solvent. ${ }^{19} \mathrm{~F}$ NMR spectra were recorded at $564 \mathrm{MHz}$ at $25{ }^{\circ} \mathrm{C}$ in $\mathrm{CDCl}_{3} / \mathrm{CD}_{3} \mathrm{OD}$. The chemical shifts of all ${ }^{1} \mathrm{H}$ NMR and ${ }^{13} \mathrm{C}$ NMR spectra were referenced to the residual signal of $\mathrm{CDCl}_{3}\left(\delta 7.26\right.$ for the ${ }^{1} \mathrm{H}$ NMR spectra and $\delta$ 77.00 for the ${ }^{13} \mathrm{C}$ NMR spectra) or $\mathrm{CD}_{3} \mathrm{OD}(\delta 3.31$ for the ${ }^{1} \mathrm{H}$ NMR spectra and $\delta 49.00$ for the ${ }^{13} \mathrm{C}$ NMR spectra). Enantiomeric excesses were determined with a Chiralcel OD-H column eluting with a mixture of hexane and propan-2-ol $(V: V=95: 5,1.0 \mathrm{~mL} / \mathrm{min}, \lambda=254 \mathrm{~nm})$. Infrared spectra (IR) were obtained with thin film samples on a PerkinElmer Spectrum Two spectrometer. HRMS were obtained from mass spectrometer (ESI-TOF). Melting points were recorded on an electrothermal digital melting point apparatus and were uncorrected. The crystal structure determination was carried out by an X-ray diffraction apparatus. Unless otherwise stated, all starting materials, catalysts, and solvents were commercially available and were used as purchased. Anhydrous solvent 1,4-dioxane, tetrahydrofuran (THF) and N,N-dimethylformamide (DMF) were used without any pretreatment. Substrates of oxabenzonorbornadienes $(\mathbf{1 a} \sim \mathbf{1 g})$ were synthesized according to the known procedures. ${ }^{[\mathrm{c}]}$ Flash column chromatography was performed using the indicated solvent system on Qingdao-Haiyang silica gel (200 300 mesh).

4.2 General procedure for the synthesis of products 3 and 4

All experiments were carried out under air. $\mathrm{PtCl}_{2}(2.7$ $\mathrm{mg}, 10 \mathrm{~mol} \%), \mathrm{PPh}_{3}(5.2 \mathrm{mg}, 20 \mathrm{~mol} \%)$, oxabicyclic alkenes 1 ( $0.1 \mathrm{mmol})$, arylsulfonyl hydrazides 2 (2 equiv., $0.2 \mathrm{mmol}), \mathrm{Cu}(\mathrm{OAc})_{2}(27.2 \mathrm{mg}, 0.15 \mathrm{mmol})$ and DCE $(2.0$ 
$\mathrm{mL}$ ) were simultaneously added to a $10 \mathrm{~mL}$ roundbottomed flask. The mixture was stirred at $80{ }^{\circ} \mathrm{C}$ in oil bath for $4 \mathrm{~h}$. After cooling to room temperature, $10 \mathrm{~mL}$ of water was added to the reaction mixture, which then was extracted by ethyl acetate $(10 \mathrm{~mL} \times 3)$. The organic layers were combined and dried with anhydrous $\mathrm{MgSO}_{4}$, and then filtered. The filtrate was concentrated under vacuum, and the resulting residue was purified by column chromatography on silica gel $(200 \sim 300$ mesh $)$ using ethyl acetate/petroleum ether or ethyl acetate/hexane to obtain the desired products 3 and 4.

$\left(1 S^{*}, 2 R^{*}\right)$-2-Phenyl-1,2-dihydronaphthalen-1-ol (3aa): Colorless oil (18.3 mg, 82\%). ${ }^{1} \mathrm{H}$ NMR (600 MHz, $\mathrm{CDCl}_{3}$ ) $\delta: 7.36 \sim 7.27(\mathrm{~m}, 6 \mathrm{H}), 7.28 \sim 7.26(\mathrm{~m}, 2 \mathrm{H}), 7.19 \sim 7.16$ $(\mathrm{m}, 1 \mathrm{H}), 6.71(\mathrm{dd}, J=9.6,2.0 \mathrm{~Hz}, 1 \mathrm{H}), 6.13(\mathrm{dd}, J=9.6$, $4.0 \mathrm{~Hz}, 1 \mathrm{H}), 4.93$ (d, $J=5.9 \mathrm{~Hz}, 1 \mathrm{H}), 3.88$ (ddd, $J=6.0$, 4.0, $2.1 \mathrm{~Hz}, 1 \mathrm{H}), 1.56(\mathrm{~s}, 1 \mathrm{H}) ;{ }^{13} \mathrm{C} \mathrm{NMR}(150 \mathrm{MHz}$, $\left.\mathrm{CDCl}_{3}\right) \delta: 137.8,136.1,132.7,129.7,129.3,128.7,128.4$, 128.3, 128.0, 127.5, 126.7, 126.4, 71.3, 47.4; IR (film) $v$ : $3445,2925,1495,1449,1128,765,691 \mathrm{~cm}^{-1}$; HRMS (ESI-TOF) calcd for $\mathrm{C}_{16} \mathrm{H}_{11} \mathrm{O}[\mathrm{M}-3 \mathrm{H}]^{-}$219.0810, found 219.0810 .

$\left(1 S^{*}, 2 R^{*}\right)$-2-( $p$-Tolyl)-1,2-dihydronaphthalen-1-ol (3ab): Colorless oil (16.1 mg, 68\%). ${ }^{1} \mathrm{H}$ NMR (600 MHz, $\left.\mathrm{CDCl}_{3}\right) \delta: 7.34(\mathrm{~d}, J=7.2 \mathrm{~Hz}, 1 \mathrm{H}), 7.30 \sim 7.22(\mathrm{~m}, 2 \mathrm{H})$, $7.17 \sim 7.10(\mathrm{~m}, 5 \mathrm{H}), 6.68(\mathrm{dd}, J=9.6,1.9 \mathrm{~Hz}, 1 \mathrm{H}), 6.11$ (dd, $J=9.6,4.1 \mathrm{~Hz}, 1 \mathrm{H}), 4.91$ (t, $J=6.6 \mathrm{~Hz}, 1 \mathrm{H}), 3.83$ (ddd, $J=6.1,4.2,2.0 \mathrm{~Hz}, 1 \mathrm{H}), 2.31$ (s, 3H), 1.49 (d, $J=7.8$ $\mathrm{Hz}, 1 \mathrm{H}) ;{ }^{13} \mathrm{C} \mathrm{NMR}\left(150 \mathrm{MHz}, \mathrm{CDCl}_{3}\right) \delta: 137.1,136.2$, $134.3,132.7,129.9,129.4,129.1,128.2,128.1,127.9$, 126.6, 126.3, 71.3, 46.9, 21.0; IR (film) v: 3437, 2920, 1512, 1451, 1374, 1184, 1071, $786 \mathrm{~cm}^{-1}$; HRMS (ESITOF) calcd for $\mathrm{C}_{17} \mathrm{H}_{13} \mathrm{O}[\mathrm{M}-3 \mathrm{H}]^{-}$233.0966, found 233.0970.

$\left(1 S^{*}, 2 R^{*}\right)$-2-(4-Methoxyphenyl)-1,2-dihydronaphthalen1-ol (3ac): Colorless oil (16.7 mg, 66\%). ${ }^{1} \mathrm{H}$ NMR $(600$ $\left.\mathrm{MHz}, \mathrm{CDCl}_{3}\right) \delta: 7.35(\mathrm{dd}, J=7.3,0.5 \mathrm{~Hz}, 1 \mathrm{H}), 7.29 \sim 7.23$ $(\mathrm{m}, 2 \mathrm{H}), 7.18 \sim 7.14(\mathrm{~m}, 3 \mathrm{H}), 6.84(\mathrm{~d}, J=8.7 \mathrm{~Hz}, 2 \mathrm{H})$, 6.68 (dd, $J=9.6,1.9 \mathrm{~Hz}, 1 \mathrm{H}), 6.10$ (dd, $J=9.6,4.3 \mathrm{~Hz}$, $1 \mathrm{H}), 4.93$ (t, $J=5.9 \mathrm{~Hz}, 1 \mathrm{H}), 3.81$ (ddd, $J=6.2,4.3,2.0$ $\mathrm{Hz}, 1 \mathrm{H}), 3.77(\mathrm{~s}, 3 \mathrm{H}), 1.49(\mathrm{~d}, J=6.9 \mathrm{~Hz}, 1 \mathrm{H}) ;{ }^{13} \mathrm{C}$ NMR $\left(150 \mathrm{MHz}, \mathrm{CDCl}_{3}\right) \delta: 159.0,136.2,132.7,130.3,130.0$, $129.1,128.2,128.0,128.0,126.5,126.3,114.1,71.3,55.2$, 46.4; IR (film) v: 3448, 2925, 1601, 1512, 1242, 1174, 1025, $796 \mathrm{~cm}^{-1}$; HRMS (ESI-TOF) calcd for $\mathrm{C}_{17} \mathrm{H}_{13} \mathrm{O}_{2}$ $[\mathrm{M}-3 \mathrm{H}]^{-}$249.0916, found 249.0916.

$\left(1 S^{*}, 2 R^{*}\right)$-2-(2-Chlorophenyl)-1,2-dihydronaphthalen-1ol (3ad): Colorless oil (15.4 mg, 60\%). ${ }^{1} \mathrm{H}$ NMR (600 $\left.\mathrm{MHz} \mathrm{CDCl}_{3}\right) \delta: 7.45(\mathrm{dd}, J=7.5,1.8 \mathrm{~Hz}, 1 \mathrm{H}), 7.42 \sim 7.34$ $(\mathrm{m}, 3 \mathrm{H}), 7.31 \sim 7.22(\mathrm{~m}, 4 \mathrm{H}), 6.76(\mathrm{dd}, J=9.6,2.7 \mathrm{~Hz}$, $1 \mathrm{H}), 6.10 \sim 6.04(\mathrm{~m}, 1 \mathrm{H}), 4.91(\mathrm{~d}, J=5.0 \mathrm{~Hz}, 1 \mathrm{H}), 4.51$ $(\mathrm{dt}, J=5.3,2.9 \mathrm{~Hz}, 1 \mathrm{H}), 1.64(\mathrm{~s}, 1 \mathrm{H}) ;{ }^{13} \mathrm{C} \mathrm{NMR}(150$ $\left.\mathrm{MHz}, \mathrm{CDCl}_{3}\right) \delta: 136.9,135.2,134.1,132.1,131.0,129.5$, $129.0,128.8,128.4,128.3,128.0,127.9,126.9,126.7$, 69.1, 44.0; IR (film) v: 3414, 2923, 1469, 1456, 1257, 1071, 1031, $808 \mathrm{~cm}^{-1}$; HRMS (ESI-TOF) calcd for $\mathrm{C}_{16} \mathrm{H}_{10} \mathrm{ClO}[\mathrm{M}-3 \mathrm{H}]^{-}$253.0418, found 253.0421. $\left(1 S^{*}, 2 R^{*}\right)$-2-(4-Chlorophenyl)-1,2-dihydronaphthalen-1ol (3ae): White solid (20.0 mg, 78\%). m.p. $113.0 \sim$ $114.0{ }^{\circ} \mathrm{C} ;{ }^{1} \mathrm{H}$ NMR $\left(600 \mathrm{MHz}, \mathrm{CDCl}_{3}\right) \delta: 7.33 \sim 7.24(\mathrm{~m}$, $5 \mathrm{H}), 7.20 \sim 7.14(\mathrm{~m}, 3 \mathrm{H}), 6.70(\mathrm{dd}, J=9.6,2.0 \mathrm{~Hz}, 1 \mathrm{H})$, $6.06(\mathrm{dd}, J=9.6,4.1 \mathrm{~Hz}, 1 \mathrm{H}), 4.89$ (d, $J=6.0 \mathrm{~Hz}, 1 \mathrm{H})$, 3.81 (ddd, $J=6.0,4.1,2.0 \mathrm{~Hz}, 1 \mathrm{H}), 1.55(\mathrm{~s}, 1 \mathrm{H}) ;{ }^{13} \mathrm{C} \mathrm{NMR}$ $\left(150 \mathrm{MHz}, \mathrm{CDCl}_{3}\right) \delta: 136.3,135.9,133.1,132.4,130.6$, 129.2, 128.6, 128.4, 128.4, 128.2, 126.5, 126.4, 71.2, 46.7; IR (film) v: 3424, 2909, 1484, 1413, 1189, 1089, 1012, 801 $\mathrm{cm}^{-1}$; HRMS (ESI-TOF) calcd for $\mathrm{C}_{16} \mathrm{H}_{10} \mathrm{ClO}[\mathrm{M}-3 \mathrm{H}]^{-}$ 253.0418, found 253.0422 .

$\left(1 S^{*}, 2 R^{*}\right)$-2-(4-Fluorophenyl)-1,2-dihydronaphthalen-1ol (3af): White solid (20.4 mg, 85\%). m.p. $100.0 \sim$ $101.0{ }^{\circ} \mathrm{C} ;{ }^{1} \mathrm{H}$ NMR $\left(600 \mathrm{MHz}, \mathrm{CDCl}_{3}\right) \delta: 7.34 \sim 7.16(\mathrm{~m}$, $6 \mathrm{H}), 7.01 \sim 6.93(\mathrm{~m}, 2 \mathrm{H}), 6.70(\mathrm{dd}, J=9.6,2.0 \mathrm{~Hz}, 1 \mathrm{H})$, $6.08(\mathrm{dd}, J=9.6,4.1 \mathrm{~Hz}, 1 \mathrm{H}), 4.91(\mathrm{t}, J=6.8 \mathrm{~Hz}, 1 \mathrm{H}), 3.84$ (ddd, $J=6.1,4.2,2.0 \mathrm{~Hz}, 1 \mathrm{H}), 1.46$ (d, $J=8.1 \mathrm{~Hz}, 1 \mathrm{H})$; ${ }^{13} \mathrm{C}$ NMR $\left(150 \mathrm{MHz}, \mathrm{CDCl}_{3}\right) \delta: 162.2(\mathrm{~d}, J=240.0 \mathrm{~Hz})$, $136.0,133.3(\mathrm{~d}, J=3.2 \mathrm{~Hz}), 132.5,130.8(\mathrm{~d}, J=8.0 \mathrm{~Hz})$, $129.6,128.4,128.3,128.1,126.5,126.4,115.4$ (d, $J=21.3$ $\mathrm{Hz}), 71.2,46.5 ;{ }^{19} \mathrm{~F}$ NMR $\left(564 \mathrm{MHz}, \mathrm{CDCl}_{3}\right) \delta:-115.4$ (s); IR (film) $v: 3420,2922,1484,1456,1410,1086,1012$, $832 \mathrm{~cm}^{-1}$; HRMS (ESI-TOF) calcd for $\mathrm{C}_{16} \mathrm{H}_{10} \mathrm{FO}$ [M$3 \mathrm{H}]^{-}$237.0713, found 237.0715.

(1 $\left.S^{*}, 2 R^{*}\right)$-2-(4-Nitrophenyl)-1,2-dihydronaphthalen-1ol (3ag): Pale-yellow solid (20.6 mg, 77\%). m.p. 122.3 $123.6{ }^{\circ} \mathrm{C} ;{ }^{1} \mathrm{H}$ NMR $\left(600 \mathrm{MHz}, \mathrm{CDCl}_{3}\right) \delta: 8.15(\mathrm{~d}, J=8.7$ $\mathrm{Hz}, 2 \mathrm{H}), 7.44$ (d, $J=8.6 \mathrm{~Hz}, 2 \mathrm{H}), 7.33$ (dd, $J=11.5,4.1$ $\mathrm{Hz}, 2 \mathrm{H}), 7.29 \sim 7.27(\mathrm{~m}, 1 \mathrm{H}), 7.21(\mathrm{~d}, J=7.3 \mathrm{~Hz}, 1 \mathrm{H})$, $6.77(\mathrm{dd}, J=9.6,2.0 \mathrm{~Hz}, 1 \mathrm{H}), 6.08(\mathrm{dd}, J=9.6,3.8 \mathrm{~Hz}$, $1 \mathrm{H}), 4.93(\mathrm{~s}, 1 \mathrm{H}), 3.95(\mathrm{t}, J=5.7 \mathrm{~Hz}, 1 \mathrm{H}), 1.60(\mathrm{~s}, 1 \mathrm{H}) ;{ }^{13} \mathrm{C}$ NMR $\left(150 \mathrm{MHz}, \mathrm{CDCl}_{3}\right) \delta: 147.2,146.5,135.6,132.1$, $130.2,129.0,128.8,128.5,128.1,126.8,126.7,123.6$, 71.2, 47.3; IR (film) v: 3417, 2922, 1591, 1513, 1339, 1107, 1071, $857 \mathrm{~cm}^{-1}$; HRMS (ESI-TOF) calcd for $\mathrm{C}_{16} \mathrm{H}_{10} \mathrm{NO}_{3}[\mathrm{M}-3 \mathrm{H}]^{-}$264.0658, found 264.0658.

$\left(1 S^{*}, 2 R^{*}\right)$-2-Mesityl-1,2-dihydronaphthalen-1-ol (3ah): Colorless oil $(13.8 \mathrm{mg}, 52 \%) .{ }^{1} \mathrm{H}$ NMR $(600 \mathrm{MHz}$, $\left.\mathrm{CD}_{3} \mathrm{OD}\right) \delta: 7.36 \sim 7.31(\mathrm{~m}, 2 \mathrm{H}), 7.26 \sim 7.19(\mathrm{~m}, 2 \mathrm{H}), 6.87$ $(\mathrm{s}, 2 \mathrm{H}), 6.61(\mathrm{dd}, J=9.6,3.3 \mathrm{~Hz}, 1 \mathrm{H}), 6.16(\mathrm{~d}, J=9.6 \mathrm{~Hz}$, $1 \mathrm{H}), 4.69$ (d, $J=4.9 \mathrm{~Hz}, 1 \mathrm{H}), 4.14 \sim 4.10(\mathrm{~m}, 1 \mathrm{H}), 2.47$ (s, $3 \mathrm{H}), 2.33(\mathrm{~s}, 3 \mathrm{H}), 2.25(\mathrm{~s}, 3 \mathrm{H}) ;{ }^{13} \mathrm{C}$ NMR $(150 \mathrm{MHz}$, $\left.\mathrm{CD}_{3} \mathrm{OD}\right) \delta$ : $140.4,137.7,137.3,136.7,135.1,134.3,133.5$, 131.8, 130.1, 129.8, 129.6, 128.1, 127.5, 125.2, 71.1, 44.8, 22.0, 21.1, 20.9; IR (film) v: 3420, 2919, 1520, 1485, 1453, 1079, 812, $761 \mathrm{~cm}^{-1}$; HRMS (ESI-TOF) calcd for $\mathrm{C}_{19} \mathrm{H}_{17} \mathrm{O}[\mathrm{M}-3 \mathrm{H}]^{-}$261.1279, found 261.1277.

1,4-Dimethoxy-6-phenylnaphthalene (4ba): Colorless oil $(17.9 \mathrm{mg}, 68 \%)$. ${ }^{1} \mathrm{H}$ NMR $\left(600 \mathrm{MHz}, \mathrm{CDCl}_{3}\right) \delta: 8.44$ $(\mathrm{d}, J=1.7 \mathrm{~Hz}, 1 \mathrm{H}), 8.27(\mathrm{~d}, J=8.7 \mathrm{~Hz}, 1 \mathrm{H}), 7.78 \sim 7.74$ $(\mathrm{m}, 3 \mathrm{H}), 7.46(\mathrm{dd}, J=10.6,4.8 \mathrm{~Hz}, 2 \mathrm{H}), 7.35(\mathrm{dt}, J=8.4$, $1.1 \mathrm{~Hz}, 1 \mathrm{H}), 6.70 \sim 6.66(\mathrm{~m}, 2 \mathrm{H}), 3.95(\mathrm{~d}, J=1.9 \mathrm{~Hz}, 6 \mathrm{H})$; ${ }^{13} \mathrm{C} \mathrm{NMR}\left(150 \mathrm{MHz}, \mathrm{CDCl}_{3}\right) \delta: 149.7,149.4,141.3,138.4$, $128.7,127.4,127.2,126.6,125.4,125.3,122.4,119.8$, 103.6, 103.3, 55.7; IR (film) v: 2940, 1599, 1462, 1364, 1272, 1229, 1097, $755 \mathrm{~cm}^{-1}$; HRMS (ESI-TOF) calcd for $\mathrm{C}_{18} \mathrm{H}_{17} \mathrm{O}_{2}[\mathrm{M}+\mathrm{H}]^{+}$265.1229, found 265.1225. 
1,4-Dimethoxy-6-( $p$-tolyl)naphthalene (4bb): White solid (18.4 mg, 66\%). m.p. 113.2 114.4 ${ }^{\circ} \mathrm{C} ;{ }^{1} \mathrm{H}$ NMR $\left(600 \mathrm{MHz}, \mathrm{CDCl}_{3}\right) \delta: 8.42(\mathrm{~d}, J=1.6 \mathrm{~Hz}, 1 \mathrm{H}), 8.25(\mathrm{~d}, J=$ $8.7 \mathrm{~Hz}, 1 \mathrm{H}), 7.75$ (dd, $J=8.7,1.9 \mathrm{~Hz}, 1 \mathrm{H}), 7.65$ (d, $J=8.1$ $\mathrm{Hz}, 2 \mathrm{H}), 7.26$ (d, $J=7.8 \mathrm{~Hz}, 2 \mathrm{H}), 6.67$ (q, $J=8.3 \mathrm{~Hz}, 2 \mathrm{H})$, $3.95(\mathrm{~d}, J=3.0 \mathrm{~Hz}, 6 \mathrm{H}), 2.40(\mathrm{~s}, 3 \mathrm{H}) ;{ }^{13} \mathrm{C}$ NMR $(150 \mathrm{MHz}$, $\left.\mathrm{CDCl}_{3}\right) \delta: 149.7,149.5,138.4,138.3,137.0,129.5,127.2$, 126.6, 125.2, 125.2, 122.4, 119.4, 103.6, 103.1, 55.7, 21.1; IR (film) v: 2937, 1594, 1520, 1459, 1359, 1267, 1232, 1104, $804 \mathrm{~cm}^{-1}$; HRMS (ESI-TOF) calcd for $\mathrm{C}_{19} \mathrm{H}_{19} \mathrm{O}_{2}$ $[\mathrm{M}+\mathrm{H}]^{+}$279.1385, found 279.1380 .

6-(4-Fluorophenyl)-1,4-dimethoxynaphthalene (4bf): White solid (22.0 mg, 78\%). m.p. 110.5 111.6 ${ }^{\circ} \mathrm{C} ;{ }^{1} \mathrm{H}$ NMR (600 MHz, $\left.\mathrm{CDCl}_{3}\right) \delta: 8.37(\mathrm{~d}, J=1.8 \mathrm{~Hz}, 1 \mathrm{H}), 8.25$ $(\mathrm{d}, J=8.7 \mathrm{~Hz}, 1 \mathrm{H}), 7.71 \sim 7.68(\mathrm{~m}, 3 \mathrm{H}), 7.14(\mathrm{t}, J=8.7$ $\mathrm{Hz}, 2 \mathrm{H}), 6.70(\mathrm{dd}, J=14.6,7.1 \mathrm{~Hz}, 2 \mathrm{H}), 3.96$ (d, $J=1.5$ $\mathrm{Hz}, 6 \mathrm{H}) ;{ }^{13} \mathrm{C} \mathrm{NMR}\left(150 \mathrm{MHz}, \mathrm{CDCl}_{3}\right) \delta: 162.5(\mathrm{~d}, J=$ $244.9 \mathrm{~Hz}), 149.2$ (d, $J=27.6 \mathrm{~Hz}), 137.4,129.0$ (d, $J=8.06$ $\mathrm{Hz}), 126.4$ (d, $J=33.1 \mathrm{~Hz}), 125.8,125.3,125.1,122.6$, $121.7,119.7,115.6(\mathrm{~d}, J=21.6 \mathrm{~Hz}), 103.7,103.2(\mathrm{~d}, J=$ $28.56 \mathrm{~Hz}$ ), 55.7; ${ }^{19} \mathrm{~F}$ NMR $\left(564 \mathrm{MHz}, \mathrm{CDCl}_{3}\right) \delta$ : -115.9 (s); IR (film) v: 2955, 1599, 1510, 1456, 1364, 1268, 1225, 1104, $827 \mathrm{~cm}^{-1}$; HRMS (ESI-TOF) calcd for $\mathrm{C}_{18} \mathrm{H}_{16} \mathrm{FO}_{2}$ $[\mathrm{M}+\mathrm{H}]^{+}$283.1134, found 283.1130.

2,3-Dimethoxy-6-phenylnaphthalene (4ca): White solid (18.5 mg, 70\%). m.p. $121.0 \sim 122.0{ }^{\circ} \mathrm{C} ;{ }^{1} \mathrm{H}$ NMR $(600$ $\left.\mathrm{MHz}, \mathrm{CDCl}_{3}\right) \delta: 7.92(\mathrm{~d}, J=1.3 \mathrm{~Hz}, 1 \mathrm{H}), 7.76(\mathrm{~d}, J=8.4$ $\mathrm{Hz}, 1 \mathrm{H}), 7.73 \sim 7.71(\mathrm{~m}, 2 \mathrm{H}), 7.62(\mathrm{dd}, J=8.4,1.8 \mathrm{~Hz}$, 1H), 7.48 (t, $J=7.7 \mathrm{~Hz}, 2 \mathrm{H}), 7.37$ (t, $J=7.5 \mathrm{~Hz}, 1 \mathrm{H}), 7.19$ (s, 1H), $7.15(\mathrm{~s}, 1 \mathrm{H}), 4.03(\mathrm{~d}, J=1.8 \mathrm{~Hz}, 6 \mathrm{H}) ;{ }^{13} \mathrm{C} \mathrm{NMR}$ $\left(150 \mathrm{MHz}, \mathrm{CDCl}_{3}\right) \delta: 149.8,149.5,141.3,136.9,129.4$, $128.7,128.3,127.2,127.0,126.8,124.3,123.8,106.5$, 106.0, 55.8; IR (film) v: 2928, 1601, 1495, 1252, 1235, $1166,1128,1003,854 \mathrm{~cm}^{-1}$; HRMS (ESI-TOF) calcd for $\mathrm{C}_{18} \mathrm{H}_{17} \mathrm{O}_{2}[\mathrm{M}+\mathrm{H}]^{+}$265.1229, found 265.1225.

2,3-Dimethoxy-6-(p-tolyl)naphthalene (4cb): White solid (20.0 mg, 72\%). m.p. 136.2 137.3 ${ }^{\circ} \mathrm{C} ;{ }^{1} \mathrm{H}$ NMR $\left(600 \mathrm{MHz}, \mathrm{CDCl}_{3}\right) \delta: 7.88(\mathrm{~d}, J=1.4 \mathrm{~Hz}, 1 \mathrm{H}), 7.73(\mathrm{~d}, J=$ $8.4 \mathrm{~Hz}, 1 \mathrm{H}), 7.60 \sim 7.58(\mathrm{~m}, 3 \mathrm{H}), 7.27(\mathrm{~d}, J=7.8 \mathrm{~Hz}, 2 \mathrm{H})$, 7.15 (d, $J=22.1 \mathrm{~Hz}, 2 \mathrm{H}), 4.01(\mathrm{~d}, J=2.2 \mathrm{~Hz}, 6 \mathrm{H}), 2.41$ (s, $3 \mathrm{H}) ;{ }^{13} \mathrm{C}$ NMR $\left(150 \mathrm{MHz}, \mathrm{CDCl}_{3}\right) \delta: 149.7,149.4,138.4$, $136.9,136.7,129.5,129.4,128.2,127.0,126.7,124.0$, $123.8,106.5,106.1,55.8,21.1$; IR (film) $v: 2948,1602$, 1456, 1360, 1268, 1232, 1097, $812 \mathrm{~cm}^{-1}$; HRMS (ESI-TOF) calcd for $\mathrm{C}_{19} \mathrm{H}_{19} \mathrm{O}_{2}[\mathrm{M}+\mathrm{H}]^{+}$279.1385, found 279.1383 .

$\left(1 S^{*}, 2 R^{*}\right)$-6,7-Dibromo-2-phenyl-1,2-dihydronaphthalen-1-ol (3da): Colorless oil (31.5 mg, 83\%). ${ }^{1} \mathrm{H}$ NMR $\left(600 \mathrm{MHz}, \mathrm{CD}_{3} \mathrm{OD}\right) \delta: 7.59(\mathrm{~s}, 1 \mathrm{H}), 7.50(\mathrm{~s}, 1 \mathrm{H}), 7.26 \sim$ $7.17(\mathrm{~m}, 5 \mathrm{H}), 6.66(\mathrm{dd}, J=9.6,1.7 \mathrm{~Hz}, 1 \mathrm{H}), 6.23(\mathrm{dd}, J=$ 9.6, $4.6 \mathrm{~Hz}, 1 \mathrm{H}), 4.93$ (d, $J=6.4 \mathrm{~Hz}, 1 \mathrm{H}), 3.78$ (ddd, $J=$ 6.4, 4.6, $1.8 \mathrm{~Hz}, 1 \mathrm{H}) ;{ }^{13} \mathrm{C} \mathrm{NMR}\left(150 \mathrm{MHz}, \mathrm{CD}_{3} \mathrm{OD}\right) \delta$ : $139.5,138.9,135.6,134.0,132.5,131.8,130.5,129.1$, 128.0, 127.0, 124.3, 123.9, 71.1, 48.2; IR (film) v: 3396, 2912, 1655, 1495, 1444, 1257, 1076, 890, $801 \mathrm{~cm}^{-1}$; HRMS (ESI-TOF) calcd for $\mathrm{C}_{16} \mathrm{H}_{9} \mathrm{Br}_{2} \mathrm{O} \quad[\mathrm{M}-3 \mathrm{H}]$ 374.9021, found 374.9024 . $\left(1 S^{*}, 2 R^{*}\right)$-6,7-Dibromo-2-( $p$-tolyl)-1,2-dihydronaphthalen-1-ol (3db)(CCDC 1966855): Colorless oil (31.4 mg, $80 \%) .{ }^{1} \mathrm{H} \mathrm{NMR}\left(600 \mathrm{MHz}, \mathrm{CDCl}_{3}\right) \delta: 7.58(\mathrm{~s}, 1 \mathrm{H}), 7.39(\mathrm{~s}$, $1 \mathrm{H}), 7.10$ (d, $J=7.9 \mathrm{~Hz}, 2 \mathrm{H}), 7.04$ (d, $J=8.0 \mathrm{~Hz}, 2 \mathrm{H}), 6.58$ (dd, $J=9.7,1.0 \mathrm{~Hz}, 1 \mathrm{H}), 6.19$ (dd, $J=9.6,4.9 \mathrm{~Hz}, 1 \mathrm{H})$, $4.95(\mathrm{~s}, 1 \mathrm{H}), 3.81 \sim 3.77(\mathrm{~m}, 1 \mathrm{H}), 2.31(\mathrm{~s}, 3 \mathrm{H}), 1.52(\mathrm{~s}$, $1 \mathrm{H}) ;{ }^{13} \mathrm{C} \mathrm{NMR}\left(150 \mathrm{MHz}, \mathrm{CDCl}_{3}\right) \delta: 137.7,137.3,133.6$, $132.2,132.0,131.4,130.7,129.6,129.1,126.2,123.9$, 123.5, 70.2, 46.2, 21.0; IR (film) v: 3417, 2915, 1510, 1464, 1260, 1107, 1072, $887 \mathrm{~cm}^{-1}$; HRMS (ESI-TOF) calcd for $\mathrm{C}_{17} \mathrm{H}_{11} \mathrm{Br}_{2} \mathrm{O}[\mathrm{M}-3 \mathrm{H}]^{-}$388.9177, found 388.9183 .

$\left(1 S^{*}, 2 R^{*}\right)-6,7-D i b r o m o-2-(4-f l u o r o p h e n y l)-1,2-d i h y d r o-$ naphthalen-1-ol (3df): Colorless oil $(35.0 \mathrm{mg}, 88 \%) .{ }^{1} \mathrm{H}$ NMR (600 MHz, CD 3 OD) $\delta: 7.59(\mathrm{~s}, 1 \mathrm{H}), 7.50(\mathrm{~s}, 1 \mathrm{H})$, $7.20 \sim 7.14(\mathrm{~m}, 2 \mathrm{H}), 6.98 \sim 6.94(\mathrm{~m}, 2 \mathrm{H}), 6.66(\mathrm{dd}, J=9.6$, $1.5 \mathrm{~Hz}, 1 \mathrm{H}), 6.21(\mathrm{dd}, J=9.6,4.7 \mathrm{~Hz}, 1 \mathrm{H}), 4.92$ (d, $J=6.4$ $\mathrm{Hz}, 1 \mathrm{H}), 3.81 \sim 3.77(\mathrm{~m}, 1 \mathrm{H}) ;{ }^{13} \mathrm{C}$ NMR $(150 \mathrm{MHz}$, $\left.\mathrm{CD}_{3} \mathrm{OD}\right) \delta: 163.5(\mathrm{~d}, J=241.7 \mathrm{~Hz}), 139.4,135.5,134.6(\mathrm{~d}$, $J=2.7 \mathrm{~Hz}), 133.8,132.4,132.2(\mathrm{~d}, J=8.0 \mathrm{~Hz}), 131.8$, 127.2, 124.3, 124.0, 115.7 (d, $J=21.6 \mathrm{~Hz}), 71.0,47.3 ;{ }^{19} \mathrm{~F}$ NMR (564 MHz, CD 3 OD) $\delta$ : -118.4 (s); IR (film) $v$ : 3414, 2915, 1596, 1510, 1464, 1219, 1155, $887 \mathrm{~cm}^{-1}$; HRMS (ESI-TOF) calcd for $\mathrm{C}_{16} \mathrm{H}_{8} \mathrm{Br}_{2} \mathrm{FO}[\mathrm{M}-3 \mathrm{H}]$ 392.8927, found 392.8932.

$\left(1 S^{*}, 2 R^{*}\right)-6,7-D i m e t h y l-2-p h e n y l-1,2-$ dihydronaphthalen-1-ol (3ea): Pale-yellow oil (17.8 mg, 71\%). ${ }^{1} \mathrm{H}$ NMR (600 MHz, CD $\left.{ }_{3} \mathrm{OD}\right) \delta: 7.25$ (dd, $J=5.9,1.2 \mathrm{~Hz}$, 4H), $7.21 \sim 7.16(\mathrm{~m}, 1 \mathrm{H}), 7.08(\mathrm{~s}, 1 \mathrm{H}), 6.94(\mathrm{~s}, 1 \mathrm{H}), 6.63$ (dd, $J=9.6,2.2 \mathrm{~Hz}, 1 \mathrm{H}), 6.02(\mathrm{dd}, J=9.6,3.8 \mathrm{~Hz}, 1 \mathrm{H})$, $4.78(\mathrm{~d}, J=5.7 \mathrm{~Hz}, 1 \mathrm{H}), 3.74$ (ddd, $J=5.9,3.8,2.3 \mathrm{~Hz}$, $1 \mathrm{H}), 2.25(\mathrm{~d}, J=5.9 \mathrm{~Hz}, 6 \mathrm{H}) ;{ }^{13} \mathrm{C} \mathrm{NMR}(150 \mathrm{MHz}$, $\left.\mathrm{CD}_{3} \mathrm{OD}\right) \delta$ : $140.9,137.1,137.0,135.4,132.0,130.6,130.2$, 129.2, 129.1, 128.9, 128.6, 127.7, 72.3, 49.1, 19.7, 19.5; IR (film) v: 3399, 2919, 1648, 1487, 1089, 1061, 1018, 887 $\mathrm{cm}^{-1}$; HRMS (ESI-TOF) calcd for $\mathrm{C}_{18} \mathrm{H}_{15} \mathrm{O}[\mathrm{M}-3 \mathrm{H}]^{-}$ 247.1124, found 247.1132 .

$\left(1 S^{*}, 2 R^{*}\right)$-2-(4-Chlorophenyl)-6,7-dimethyl-1,2-dihydronaphthalen-1-ol (3ee): Colorless oil $(20.5 \mathrm{mg}, 72 \%) .{ }^{1} \mathrm{H}$ NMR (600 MHz, CD 3 OD) $\delta: 7.25 \sim 7.21(\mathrm{~m}, 4 \mathrm{H}), 7.09(\mathrm{~s}$, $1 \mathrm{H}), 6.94(\mathrm{~s}, 1 \mathrm{H}), 6.64$ (dd, $J=9.6,1.9 \mathrm{~Hz}, 1 \mathrm{H}), 5.99$ (dd, $J=9.6,4.0 \mathrm{~Hz}, 1 \mathrm{H}), 4.80$ (d, $J=5.9 \mathrm{~Hz}, 1 \mathrm{H}), 3.74$ (ddd, $J=6.0,4.0,2.1 \mathrm{~Hz}, 1 \mathrm{H}), 2.26(\mathrm{~d}, J=5.0 \mathrm{~Hz}, 6 \mathrm{H}) ;{ }^{13} \mathrm{C}$ NMR $\left(150 \mathrm{MHz}, \mathrm{CD}_{3} \mathrm{OD}\right) \delta: 139.6,137.2,137.1,135.2$, $133.4,132.1,131.8,129.6,129.2,129.1,129.0,128.6$, 72.0, 48.3, 19.7, 19.5; IR (film) v: 3414, 2920, 1510, 1489, 1311, 1253, 1094, $887 \mathrm{~cm}^{-1}$; HRMS (ESI-TOF) calcd for $\mathrm{C}_{18} \mathrm{H}_{14} \mathrm{ClO}[\mathrm{M}-3 \mathrm{H}]^{-}$281.0734, found 281.0738.

$\left(1 S^{*}, 2 R^{*}\right)-6,7-D i m e t h y l-2-(4-n i t r o p h e n y l)-1,2-d i h y d r o-$ naphthalen-1-ol (3eg): Colorless oil $(20.7 \mathrm{mg}, 70 \%) .{ }^{1} \mathrm{H}$ NMR (600 MHz, $\left.\mathrm{CDCl}_{3}\right) \delta: 8.17(\mathrm{~d}, J=8.5 \mathrm{~Hz}, 2 \mathrm{H}), 7.46$ $(\mathrm{d}, J=8.5 \mathrm{~Hz}, 2 \mathrm{H}), 7.10(\mathrm{~s}, 1 \mathrm{H}), 6.99(\mathrm{~s}, 1 \mathrm{H}), 6.71(\mathrm{~d}, J=$ $8.3 \mathrm{~Hz}, 1 \mathrm{H}), 6.00$ (dd, $J=9.5,3.5 \mathrm{~Hz}, 1 \mathrm{H}), 4.86$ (d, $J=5.4$ $\mathrm{Hz}, 1 \mathrm{H}), 3.93$ (s, 1H), 2.27 (d, $J=9.6 \mathrm{~Hz}, 6 \mathrm{H}), 1.59$ (s, $1 \mathrm{H}) ;{ }^{13} \mathrm{C}$ NMR $\left(150 \mathrm{MHz}, \mathrm{CDCl}_{3}\right) \delta: 147.1,147.0,137.0$, $136.9,133.0,130.2,129.7,128.9,128.2,128.1,127.0$, 123.5, 71.1, 47.6, 19.7, 19.5; IR (film) v: 3427, 2925, 
1591, 1512, 1342, 1306, 1257, 1104, $850 \mathrm{~cm}^{-1}$; HRMS (ESI-TOF) calcd for $\mathrm{C}_{18} \mathrm{H}_{14} \mathrm{NO}_{3}[\mathrm{M}-3 \mathrm{H}]^{-}$292.0974, found 292.0982 .

$\left(1 S^{*}, 2 R^{*}\right)-6,7-D i f l u o r o-2-p h e n y l-1,2-d i h y d r o n a p h-$ thalen-1-ol (3fa): Yellow oil (22.4, 87\%). ${ }^{1} \mathrm{H}$ NMR (600 $\left.\mathrm{MHz}, \mathrm{CDCl}_{3}\right) \delta: 7.33 \sim 7.28(\mathrm{~m}, 3 \mathrm{H}), 7.21 \sim 7.17(\mathrm{~m}, 3 \mathrm{H})$, $6.98(\mathrm{dd}, J=10.5,7.6 \mathrm{~Hz}, 1 \mathrm{H}), 6.60(\mathrm{dd}, J=9.7,1.7 \mathrm{~Hz}$, $1 \mathrm{H}), 6.16(\mathrm{dd}, J=9.6,4.5 \mathrm{~Hz}, 1 \mathrm{H}), 4.95(\mathrm{~d}, J=6.4 \mathrm{~Hz}$, $1 \mathrm{H}), 3.84(\mathrm{t}, J=4.8 \mathrm{~Hz}, 1 \mathrm{H}), 1.56(\mathrm{~s}, 1 \mathrm{H}) ;{ }^{13} \mathrm{C}$ NMR $(150$ $\left.\mathrm{MHz}, \mathrm{CDCl}_{3}\right) \delta: 150.7(\mathrm{dd}, J=26.5,12.8 \mathrm{~Hz}), 149.1$ (dd, $J=28.5,12.9 \mathrm{~Hz}), 136.1,133.2$ (t, $J=4.1 \mathrm{~Hz}), 130.4$ (d, $J=2.4 \mathrm{~Hz}), 129.5(\mathrm{dd}, J=6.2,4.1 \mathrm{~Hz}), 129.3,128.8$, 127.8, 126.5, 116.0 (d, $J=18.5 \mathrm{~Hz}), 115.0(\mathrm{~d}, J=17.6 \mathrm{~Hz})$, 70.4, 46.6; ${ }^{19} \mathrm{~F}$ NMR $\left(564 \mathrm{MHz}, \mathrm{CDCl}_{3}\right) \delta:-138.3(\mathrm{~d}$, $J=21.5 \mathrm{~Hz}$ ), $-139.8(\mathrm{~d}, J=20.3 \mathrm{~Hz}$ ); IR (film) $v: 3424$, 2919, 1591, 1502, 1374, 1306, 1071, $875 \mathrm{~cm}^{-1}$; HRMS (ESI-TOF) calcd for $\mathrm{C}_{16} \mathrm{H}_{9} \mathrm{~F}_{2} \mathrm{O}[\mathrm{M}-3 \mathrm{H}]^{-}$255.0622, found 255.0626 .

$\left(1 S^{*}, 2 R^{*}\right)$-6,7-Difluoro-2-( $p$-tolyl)-1,2-dihydronaphthalen-1-ol (3fb): Colorless oil (21.8 mg, 80\%). ${ }^{1} \mathrm{H}$ NMR $\left(600 \mathrm{MHz}, \mathrm{CDCl}_{3}\right) \delta: 7.18(\mathrm{dd}, J=10.5,8.0 \mathrm{~Hz}, 1 \mathrm{H}), 7.10$ (dd, $J=23.9,8.0 \mathrm{~Hz}, 4 \mathrm{H}), 6.97$ (dd, $J=10.5,7.7 \mathrm{~Hz}, 1 \mathrm{H})$, $6.58(\mathrm{dd}, J=9.7,1.3 \mathrm{~Hz}, 1 \mathrm{H}), 6.14(\mathrm{dd}, J=9.7,4.6 \mathrm{~Hz}$, $1 \mathrm{H}), 4.94(\mathrm{~s}, 1 \mathrm{H}), 3.80(\mathrm{t}, J=5.3 \mathrm{~Hz}, 1 \mathrm{H}), 2.32(\mathrm{~s}, 3 \mathrm{H})$, $1.50(\mathrm{~s}, 1 \mathrm{H}) ;{ }^{13} \mathrm{C}$ NMR $\left(150 \mathrm{MHz}, \mathrm{CDCl}_{3}\right) \delta: 150.6(\mathrm{dd}$, $J=20.2,13.2 \mathrm{~Hz}), 149.0(\mathrm{dd}, J=22.1,12.9 \mathrm{~Hz}), 137.6$, $133.3(\mathrm{t}, J=4.5 \mathrm{~Hz}), 132.7,130.7,130.7,129.6,129.1$, 126.4, 115.9 (d, $J=18.5 \mathrm{~Hz}), 114.9$ (d, $J=18.0 \mathrm{~Hz}), 70.3$, 46.1, 21.0; ${ }^{19} \mathrm{~F}$ NMR (564 MHz, $\left.\mathrm{CDCl}_{3}\right) \delta$ : -138.4 (d, $J=20.3 \mathrm{~Hz}$ ), -140.0 (d, $J=20.3 \mathrm{~Hz}$ ); IR (film) $v: 3410$, 2923, 1507, 1449, 1378, 1308, 1072, $883 \mathrm{~cm}^{-1}$; HRMS (ESI-TOF) calcd for $\mathrm{C}_{17} \mathrm{H}_{11} \mathrm{~F}_{2} \mathrm{O}[\mathrm{M}-3 \mathrm{H}]^{-}$269.0779, found 269.0785 .

$\left(1 S^{*}, 2 R^{*}\right)-6,7-D i f l u o r o-2-(4-f l u o r o p h e n y l)-1,2-d i h y-$ dronaphthalen-1-ol (3ff): Colorless oil (24.6 mg, 89\%). ${ }^{1} \mathrm{H}$ NMR $\left(600 \mathrm{MHz}, \mathrm{CDCl}_{3}\right) \delta: 7.21 \sim 7.12(\mathrm{~m}, 3 \mathrm{H}), 7.04 \sim$ $6.94(\mathrm{~m}, 3 \mathrm{H}), 6.59$ (dd, $J=9.6,1.3 \mathrm{~Hz}, 1 \mathrm{H}), 6.12(\mathrm{dd}, J=$ 9.6, $4.6 \mathrm{~Hz}, 1 \mathrm{H}), 4.92(\mathrm{~s}, 1 \mathrm{H}), 3.80$ (t, $J=5.0 \mathrm{~Hz}, 1 \mathrm{H}), 1.51$ $(\mathrm{s}, 1 \mathrm{H}) ;{ }^{13} \mathrm{C} \mathrm{NMR}\left(150 \mathrm{MHz}, \mathrm{CDCl}_{3}\right) \delta: 162.4(\mathrm{~d}, J=244.7$ $\mathrm{Hz}), 150.7$ (dd, $J=19.2,12.8 \mathrm{~Hz}), 149.0$ (dd, $J=20.6,13.1$ $\mathrm{Hz}), 133.0$ (t, $J=4.1 \mathrm{~Hz}), 131.8(\mathrm{~d}, J=3.2 \mathrm{~Hz}), 130.8$ (d, $J=7.8 \mathrm{~Hz}), 130.3$ (d, $J=2.3 \mathrm{~Hz}), 129.4(\mathrm{dd}, J=6.2,4.0$ $\mathrm{Hz}), 126.6,115.9$ (d, $J=18.5 \mathrm{~Hz}), 115.6$ (d, $J=20.9 \mathrm{~Hz})$, $115.1(\mathrm{~d}, J=17.9 \mathrm{~Hz}), 70.3,45.8 ;{ }^{19} \mathrm{~F}$ NMR (564 MHz, $\left.\mathrm{CDCl}_{3}\right) \delta$ : $-114.7(\mathrm{~s}),-138.0(\mathrm{~d}, J=20.3 \mathrm{~Hz}),-139.5$ (d, $J=20.3 \mathrm{~Hz}$ ); IR (film) $v$ : 3424, 2922, 1597, 1505, $1313,1221,1104,878 \mathrm{~cm}^{-1}$; HRMS (ESI-TOF) calcd for $\mathrm{C}_{16} \mathrm{H}_{8} \mathrm{~F}_{3} \mathrm{O}[\mathrm{M}-3 \mathrm{H}]^{-}$273.0528, found 273.0534.

(1 $\left.S^{*}, 2 R^{*}\right)$-2-Phenyl-1,2-dihydrotriphenylen-1-ol (3ga): White solid (16.8 mg, 52\%). m.p. 156 158 ${ }^{\circ} \mathrm{C} ;{ }^{1} \mathrm{H}$ NMR $\left(600 \mathrm{MHz}, \mathrm{CDCl}_{3}\right) \delta: 8.80 \sim 8.69(\mathrm{~m}, 2 \mathrm{H}), 8.35 \sim 8.30(\mathrm{~m}$, $1 \mathrm{H}), 8.29 \sim 8.24(\mathrm{~m}, 1 \mathrm{H}), 7.73 \sim 7.59(\mathrm{~m}, 5 \mathrm{H}), 7.55(\mathrm{dd}$, $J=8.0,0.9 \mathrm{~Hz}, 2 \mathrm{H}), 7.50 \sim 7.46(\mathrm{~m}, 2 \mathrm{H}), 7.42 \sim 7.37(\mathrm{~m}$, $1 \mathrm{H}), 6.49$ (ddd, $J=9.8,2.3,1.5 \mathrm{~Hz}, 1 \mathrm{H}), 5.44(\mathrm{~s}, 1 \mathrm{H})$, $4.07 \sim 4.00(\mathrm{~m}, 1 \mathrm{H}), 1.56(\mathrm{~s}, 1 \mathrm{H}) ;{ }^{13} \mathrm{C} \mathrm{NMR}(150 \mathrm{MHz}$, $\left.\mathrm{CDCl}_{3}\right) \delta: 140.1,130.8,130.6,130.6,129.8,129.2,128.8$ $128.7,128.6,127.3,127.2,126.9,126.8,126.5,126.4$,
124.1, 123.9, 123.8, 123.1, 123.1, 67.6, 48.1; IR (film) v: 3486, 2996, 1525, 1510, 1108, 775, $710 \mathrm{~cm}^{-1}$; HRMS (ESI-TOF) calcd for $\mathrm{C}_{24} \mathrm{H}_{15} \mathrm{O}[\mathrm{M}-3 \mathrm{H}]^{-}$319.1124, found 319.1125 .

\subsection{Procedure for the scaled-up synthesis of product 3aa}

The experiment was carried out under air. $\mathrm{PtCl}_{2}(0.054$ g, $10 \mathrm{~mol} \%), \mathrm{PPh}_{3}(0.105 \mathrm{~g}, 20 \mathrm{~mol} \%)$, oxabicyclic alkenes $\mathbf{1 a}(0.288 \mathrm{~g}, 2.0 \mathrm{mmol})$, arylsulfonyl hydrazides 2a $(0.689 \mathrm{~g}, 4.0 \mathrm{mmol}), \mathrm{Cu}(\mathrm{OAc})_{2}(0.544 \mathrm{~g}, 3.0 \mathrm{mmol})$ and DCE $(20.0 \mathrm{~mL})$ were simultaneously added to a $50 \mathrm{~mL}$ round-bottomed flask. The mixture was stirred at $80{ }^{\circ} \mathrm{C}$ in oil bath for $4 \mathrm{~h}$. After cooling to room temperature, $30 \mathrm{~mL}$ of water was added to the reaction mixture, which then was extracted by ethyl acetate $(20 \mathrm{~mL} \times 3)$, The organic layers were combined and dried with anhydrous $\mathrm{MgSO}_{4}$, and then filtered. The filtrate was concentrated under vacuum, and there sulting residue was purified on silica gel [eluent: $V($ ethyl acetate $): V$ (petroleum ether $)=1: 20]$ to obtain product 3aa as a colorless oil $(0.302 \mathrm{~g}, 68 \%)$.

Supporting Information The ${ }^{1} \mathrm{H}$ NMR, ${ }^{13} \mathrm{C}$ NMR spectra of compounds 3 and $\mathbf{4}$, the ${ }^{19} \mathrm{~F}$ NMR spectra of compounds 3af, 3df, 3fa, 3fb, 3ff, and $\mathbf{4 b f}$, HPLC chromatograms of compound 3aa, and the X-ray single crystal diffraction analysis of compound $\mathbf{3 d b}$. The Supporting Information is available free of charge via the Internet at http://sioc-journal.cn/.

\section{References}

[1] (a) Ward, R. S. Chem. Soc. Rev. 1990, 19, 1.

(b) Fagnou, K.; Lautens, M. Chem. Rev. 2003, 103, 169

(c) Hayashi, T.; Yamasaki, K. Chem. Rev. 2003, 103, 2829.

(d) Lautens, M.; Fagnou, K.; Hiebert, S. Acc. Chem. Res. 2003, 36, 48.

[2] (a) Lautens, M.; Fagnou, K.; Zunic, V. Org. Lett. 2002, 4, 3465.

(b) McManus, H. A.; Fleming, M. J.; Lautens, M. Angew. Chem., Int. Ed. 2007, 46, 433.

(c) Tsoung, J.; Krämer, K.; Zajdlik, A.; Liébert, C.; Lautens, M. J. Org. Chem. 2011, 76, 9031.

[3] (a) Yang, D.-Q.; Long,Y.-H.; Wang, H.; Zhang, Z.-M. Org. Lett. 2008, 10, 4723.

(b) Long, Y.-H.; Li, X.-L.; Pan, X.-J.; Ding, D.-D.; Xu, X.; Zuo, X.-J.; Yang, D.-Q.; Wang, S.-Y.; Li, C.-R. Catal. Lett. 2014, 144, 419.

(c) Yang, W.; Luo, R.-S.; Yang, D.-Q. Molecules 2015, 20, 21103. (d) Zhu, M.-N.; Chen, J.-C.; He, X.-B.; Gu, C.-P.; Xu, J.-B.; Fan, B.-M. J. Org. Chem. 2017, 82, 3167.

(e) Yang, X.; Yang, W.; Yao, Y.-Q.; Deng, Y.-Y.; Zuo, X.-J.; Yang, D.-Q. J. Org. Chem. 2018, 83, 10097.

(f) Yang, X.; Yang, W.; Yao, Y.-Q.; Deng, Y.-Y.; Yang, D.-Q. Org. Chem. Front. 2019, 6, 1151.

[4] (a) Rayabarapu, D. K.; Chiou, C.-F.; Cheng, C.-H. Org. Lett. 2002, 4, 1679.

(b) Li, L.-P. Rayabarapu, D. K.; Nandi, M.; Cheng, C.-H. Org. Lett. 2003, 5, 1621.

(c) Mannathana, S.; Cheng, C.-H. Adv. Synth. Catal. 2014, 356, 2239.

(d) Shukla, P.; Sharma, A.; Pallavi, B.; Cheng, C.-H. Tetrahedron 2015, 71, 2260 . 
[5] (a) Cabrera, S.; Arrayás, R. G.; Carretero, J. C. Angew. Chem., Int. Ed. 2004, 43, 3944.

(b) Zhang, T.-K.; Mo, D.-L.; Dai, L.-X.; Hou, X.-L. Org. Lett. 2008, 10, 3689 .

(c) Liu, S.-S.; Li, S.-F.; Chen, H.-L.; Yang, Q.-J.; Xu, J.-B.; Zhou, Y.-Y.; Yuan, M.-L.; Zeng, W.-M.; Fan, B.-M. Adv. Synth. Catal. 2014, 356, 2960.

(d) Li, S.-F.; Xu, J.-B.; Fan, B.-M.; Lu, Z.-W.; Zeng, C.-Y.; Bian, Z.-X.; Zhou, Y.-Y.; Wang, J. Chem.-Eur. J. 2015, 21, 9003.

(e) Lu, Z.-W.; Wang, J.; Han, B.-Q.; Li, S.-F.; Zhou, Y.-Y.; Fan, B.-M. Adv. Synth. Catal. 2015, 357, 3121

(f) Zhou, H.; Li, J.-X.; Yang, H.-M.; Xia, C.-G.; Jiang, G.-X. Org. Lett. 2015, 17, 4628.

(g) Yang, F.; Chen, J.-C.; Xu, J.-B.; Ma, F.-J.; Zhou, Y.-Y.; Shinde, M. V.; Fan, B.-M. Org. Lett. 2016, 18, 4832.

[6] (a) Bertozzi, F.; Pineschi, M.; Macchia, F.; Arnold, L. A.; Minnaard, A. J.; Feringa, B. L. Org. Lett. 2002, 4, 2703.

(b) Arrayas, R. G.; Cabrera, S.; Carretero, J. C. Org. Lett. 2005, 7, 219.

(c) Zhang, W.; Wang, L.-X.; Shi, W.-J.; Zhou, Q.-L. J. Org. Chem. 2005, 70, 3734 .

(d) Zhang, W.; Zhu, S.-F.; Qiao, X.-C.; Zhou, Q.-L. Chem.-Asian J. 2008, 3, 2105 .

(e) Millet, R.; Bernardez, T.; Palais, L.; Alexakis, A. Tetrahedron Lett. 2009, 50, 3474.

(f) Millet, R.; Gremaud, L.; Bernardez, T.; Palais, L.; Alexakis, A. Synthesis 2009, 12, 2101.

(g) Bos, P. H.; Rudolph, A.; Pérez, M.; Fañanás-Mastral, M.; Harutyunyan, S. R.; Feringa, B. L. Chem. Commun. 2012, 48, 1748.

[7] (a) Leong, P.; Lautens, M. J. Org. Chem. 2004, 69, 2194.

(b) Cho, Y.-H.; Zunic, V.; Senboku, H.; Olsen, M.; Lautens, M. J. Am. Chem. Soc. 2006, 128, 6837.

(c) Nishimura, T.; Tsurumaki, E.; Kawamoto, T.; Guo, X.-X.; Hayashi, T. Org. Lett. 2008, 10, 4057.

(d) Tsui, G. C.; Lautens, M. Angew. Chem., Int. Ed. 2012, 51, 5400.

(e) Zhu, J.-T.; Tsui, G. C.; Lautens, M. Angew. Chem., Int. Ed. 2012, $51,12353$.

(f) Tsui, G. C.; Ninnemann, N. M.; Hosotani, A.; Lautens, M. Org. Lett. 2013, 5, 1064.

(g) Zhang, L.; Le, C. M.; Lautens, M. Angew. Chem., Int. Ed. 2014, 53,5951 .

(h) Chen, J.-C.; Zou, L.-L.; Zeng, C.-Y.; Zhou, Y.-Y.; Fan, B.-M. Org. Lett. 2018, 20, 1283.

[8] (a) Meng, L.; Yang, W.; Pan, X.-J.; Tao, M.; Cheng, G.; Wang, S.-Y.; Zeng, H.-P.; Long, Y.-H.; Yang, D.-Q. J. Org. Chem. 2015,
$80,2503$.

(b) Pan, X.-J.; Huang, G.-B.; Long, Y.-H.; Zuo, X.-J.; Xu, X.; Gu, F.-L.; Yang, D.-Q. J. Org. Chem. 2014, 79, 187.

[9] (a) Villeneuve, K.; Tam, W. J. Am. Chem. Soc. 2006, 128, 3514.

(b) Tenaglia, A.; Marc, S.; Giordano, L.; De Riggi, I. Angew. Chem., Int. Ed. 2011, 50, 9062.

[10] Ito, S.; Itoh, T.; Nakamura, M. Angew. Chem., Int. Ed. 2011, 50, 454.

[11] (a) Sawama, Y.; Kawamoto, K.; Satake, H.; Krause, N.; Kita, Y. Synlett 2010, 2151

(b) Huang, Y.; Ma, C.; Lee, Y. X.; Huang, R.-Z.; Zhao, Y. Angew Chem., Int. Ed. 2015, 54, 13696.

[12] (a) Zeng, C.-Y.; Yang, F.; Chen, J.-C.; Wang, J.; Fan, B.-M. Org Biomol. Chem. 2015, 13, 8425 .

(b) Xu, X.; Chen, J.-C.; He, Z.-X.; Zhou, Y.-Y.; Fan, B.-M. Org Biomol. Chem. 2016, 14, 2480.

(c) Yang, W.; Cheng, G.; Li, Y.; Zuo, X.-J.; Yang, D.-Q. Synthesis 2017, 49, 2025.

(d) Shen, G.-L.; Khan, R.; Lv, H.-P.; Yang, Y.; Zhang, X.; Zhan, Y.; Zhou, Y.-Y.; Fan, B.-M. Org. Chem. Front. 2019, 6, 1423.

[13] (a) Cheng, H.-C.; Yang, D.-Q. J. Org. Chem. 2012, 77, 9756.

(b) Fang, S.; Liang, X.-L.; Long, Y.-H.; Li, X.-L.; Yang, D.-Q.; Wang, S.-Y.; Li, C.-R. Organometallics 2012, 31, 3113.

[14] Yang, D.-Q.; Xia, J.-Y.; Long, Y.-H.; Zeng, Z.-Y.; Zuo, X.-J.; Wang, S.-Y.; Li, C.-R. Org. Biomol. Chem. 2013, 11, 4871.

[15] (a) Yang, D.-Q.; Liang, N. Org. Biomol. Chem. 2014, 12, 2080. (b) Chen, G.; Yang, W.; Li, Y.; Yang, D.-Q. J. Org. Chem. 2016, 81, 7817.

[16] Deng, Y.-Y.; Yang, W.; Yao, Y.-Q.; Yang, X.; Zuo, X.-J.; Yang, D.-Q. Org. Biomol. Chem. 2019, 17, 703.

[17] (a) Li, Y.; Yang, W.; Cheng, G.; Yang, D.-Q. J. Org. Chem. 2016, $81,4744$.

(b) Wu, R.-H.; Yang, W.; Chen, W.-K.; Yang, D.-Q. Org. Chem. Front. 2017, 4, 1921.

[18] (a) Zeng, Z.-Y.; Yang, D.-Q.; Long,Y.-H.; Pan, X.-J.; Huang, G.-B.; Zuo, X.-J.; Zhou, W. J. Org. Chem. 2014, 79, 5249.

(b) Zhang, W.; Chen, J.-C.; Zeng, G.-Z.; Yang, F.; Xu, J.-B.; Sun,W.-Q.; Shinde, M. V.; Fan, B.-M. J. Org. Chem. 2017, 82, 2641.

[19] Myers, A. G.; Zheng, B.; Movassaghi, M. J. Org. Chem. 1997, 62, 7507.

[20] Aziz, J.; Messaoudi, S.; Alami, M.; Hamze, A. Org. Biomol. Chem. 2014, 12, 9743.

[21] Chen, D.-H.; Yao, Y.-Q.; Yang, W.; Lin, Q.-F.; Li, H.-Y.; Wang, L.; Chen, S.-Q.; Tan, Y.; Yang, D.-Q. J. Org. Chem. 2019, 84, 12481.

(Cheng, F.) 